\title{
Adaptive Inverse Optimal Control of a Novel Fractional-Order Four-Wing Hyperchaotic System with Uncertain Parameter and Circuitry Implementation
}

\author{
Chaojun Wu, ${ }^{1,2}$ Gangquan Si, ${ }^{1,2}$ Yanbin Zhang, ${ }^{1,2}$ and Ningning Yang ${ }^{1,2}$ \\ ${ }^{1}$ State Key Laboratory of Electrical Insulation and Power Equipment, Xian Jiaotong University, Xian 710049, China \\ ${ }^{2}$ School of Electrical Engineering, Xian Jiaotong University, Xian 710049, China \\ Correspondence should be addressed to Gangquan Si; sigangquan@mail.xjtu.edu.cn
}

Received 12 March 2014; Revised 19 June 2014; Accepted 11 August 2014

Academic Editor: Xuejun Xie

Copyright (C) 2015 Chaojun Wu et al. This is an open access article distributed under the Creative Commons Attribution License, which permits unrestricted use, distribution, and reproduction in any medium, provided the original work is properly cited.

An efficient approach of inverse optimal control and adaptive control is developed for global asymptotic stabilization of a novel fractional-order four-wing hyperchaotic system with uncertain parameter. Based on the inverse optimal control methodology and fractional-order stability theory, a control Lyapunov function (CLF) is constructed and an adaptive state feedback controller is designed to achieve inverse optimal control of a novel fractional-order hyperchaotic system with four-wing attractor. Then, an electronic oscillation circuit is designed to implement the dynamical behaviors of the fractional-order four-wing hyperchaotic system and verify the satisfactory performance of the controller. Comparing with other fractional-order chaos control methods which may have more than one nonlinear state feedback controller, the inverse optimal controller has the advantages of simple structure, high reliability, and less control effort that is required and can be implemented by electronic oscillation circuit.

\section{Introduction}

Fractional calculus is a classical mathematical theory with history of more than 300 years. Fractional-order differential equations can describe many systems in the real world more adequately, such as electrical circuits [1-3], polymer material [4], finance systems [5], and population models [6].

Comparing with integer-order chaotic systems which exhibit complex nonlinear phenomena, fractional-order and multi-wing chaotic systems exhibit more complex and richer dynamical behaviors. It is expected that those chaotic systems will have a certain theoretical and practical significance for secure communication, control processing, and some other engineering applications. There exist some well-known fractional-order systems and multi-wing systems, such as the fractional-order Chua's circuit [7], the fractional-order Rössler system [8], the fractional-order Chen system [9],the fractional-order Lu system [10], the first true four-wing attractor [11], a family of hyperchaotic systems with four-wing attractor [12], among many others [13-22].
The applications of fractional-order differential equations in control processing developed rapidly in the last two decades. Fractional-order control methods and researches on the stability of fractional-order systems have become the frontier problem in modern nonlinear dynamics [2325]. Podlubny and his colleagues proposed the fractionalorder proportional-integral (PI) and proportional-integralderivative (PID) controllers which are named $\mathrm{PI}^{\lambda}$ and $\mathrm{PI}^{\lambda} \mathrm{D}^{\mu}$ controllers with the orders $\lambda$ and $\mu$ [26] and designed analogue circuits to implement fractional-order controllers [27]. Hamamci presented a method to stabilize a given fractional dynamic system using fractional-order $\mathrm{PI}^{\lambda}$ and $\mathrm{PI}^{\lambda} \mathrm{D}^{\mu}$ controllers [28]. In [29], the author proposed a solution scheme for a class of fractional optimal control problems. In [30], authors designed a fractional-order sliding mode controller to stabilize a fractional-order hyperchaotic system.

Nonlinear controllers have been adopted in many fields in spite of having the complex structure and being not easy to obtain. Optimal control guarantees several desirable properties for the closed-loop system, including stability margins 
and robustness. To circumvent the task of solving a HamiltonJacobi-Bellman equation whose solution is nonexistent or nonunique in generally, the inverse optimal control technique based on input-to-state stability concept was developed [31].

In this article, based on inverse optimal control methodology and fractional-order stability theory, we construct an adaptive state feedback controller to achieve the global asymptotic stabilization of a novel fractional-order fourwing hyperchaotic system. An electronic oscillation circuit is designed to realize the dynamical behaviors of the fractionalorder system and verify the satisfactory performance of the controller. Comparing with other control methods, for example, feedback control [32], active control [33], impulsive control [34], back-stepping control [35], and sliding mode control [36], the inverse optimal controller has the advantages of simple structure, high reliability, and less control effort that is required.

The rest of this paper is organized as follows. In Section 2, the inverse optimal control methodology and fractionalorder stability theory are introduced. In Section 3, firstly, a novel pseudo four-wing hyperchaotic system is analyzed. Then a derivative fractional-order four-wing hyperchaotic system is implemented by numerical simulations, and the adaptive inverse optimal control is applied to stabilize an unstable equilibrium point of the fractional-order four-wing hyperchaotic system. In Section 4, circuitry implementations are given for verifying the feasibility. Finally, the conclusion part summarizes the whole development process and presents some concluding remarks and comments.

\section{Preliminaries}

The inverse optimality approach of fractional-order fourwing hyperchaotic system used in this paper requires the knowledge of control Lyapunov function and fractionalorder stability theory.

2.1. Inverse Optimal Control Approach. Consider the following nonlinear system:

$$
\dot{x}=f(x)+g(x) u,
$$

where $x \in \mathbb{R}^{n}$ denotes the state vector and $u \in \mathbb{R}^{m}$ denotes the control vector, respectively. Moreover, $f: \mathbb{R}^{n} \rightarrow \mathbb{R}^{n}$ is vector function with $f(0)=0$ and $g: \mathbb{R}^{n} \rightarrow \mathbb{R}^{n \times m}$ is matrix-valued function.

Definition 1 (see [37]). $V(x)$ is a smooth, positive definite, and radially unbounded function. Taking the time derivative of $V(x)$, one obtains

$$
\dot{V}(x)=\frac{\partial V(x)}{\partial x}(f(x)+g(x) u)=L_{f} V+u L_{g} V,
$$

where

$$
\begin{aligned}
& L_{f} V=\frac{\partial V(x)}{\partial x} f(x), \\
& L_{g} V=\frac{\partial V(x)}{\partial x} g(x) .
\end{aligned}
$$

For all $x \neq 0, V(x)$ is a control Lyapunov function (CLF) for system (1), if it satisfies

$$
L_{g} V=0 \Longrightarrow L_{f} V<0 .
$$

Lemma 2 (see [38]). Suppose that the static state feedback control law

$$
u=k(x):=R(x)^{-1}\left(\frac{\partial V}{\partial x} g(x)\right)^{T},
$$

where $R: \mathbb{R}^{n} \rightarrow \mathbb{R}^{n \times m}$ is a positive definite matrix-valued function, stabilizes system (1) with respect to a positive definite radially unbounded Lyapunov function $V(x)$. Then the control law

$$
u:=-\beta k(x)=-\beta R^{-1}(x)\left(\frac{\partial V}{\partial x} g(x)\right)^{T}
$$

is optimal with respect to the cost

$$
J(u)=\int_{0}^{\infty}\left(l(x)+u^{T} R(x) u\right) d t
$$

where

$$
\begin{aligned}
l(x)= & -2 \beta \frac{\partial V}{\partial x}(f(x)+g(x) u) \\
& +\beta(\beta-2) \frac{\partial V}{\partial x} g(x) R^{-1}(x)\left(\frac{\partial V}{\partial x} g(x)\right)^{T} .
\end{aligned}
$$

2.2. The Fractional-Order Stability Theory. In the theory of fractional calculus, ${ }_{a} D_{t}^{\alpha}$ represents an arbitrary order differintegral operator. It is a notation for taking both fractional integrals and derivatives in one single expression and can be defined as

$$
{ }_{a} D_{t}^{\alpha}= \begin{cases}\frac{d^{\alpha}}{d t^{\alpha}}, & \alpha>0 \\ 1, & \alpha=0 \\ \int_{a}^{t}(d \tau)^{-\alpha}, & \alpha<0\end{cases}
$$

where $\alpha \in \mathbb{R}$ is the order of the operation $a$ and $t$ are the bounds of the operation.

There are some different definitions for fractional derivatives [39]. The most frequently used definitions are RiemannLiouville definition and Caputo definition.

Riemann-Liouville definition is given as

$$
\frac{d^{\alpha} f(t)}{d t^{\alpha}}=\frac{1}{\Gamma(n-\alpha)} \frac{d^{n}}{d t^{n}} \int_{a}^{t} \frac{f(\tau)}{(t-\tau)^{\alpha-n+1}} d \tau,
$$

where $n-1<\alpha<n, n$ is an integer number, and $\Gamma(\cdot)$ is the Gamma function.

If all the initial conditions are zero, the Laplace transform of Riemann-Liouville functional derivative is given as

$$
L\left\{\frac{d^{\alpha} f(t)}{d t^{\alpha}}\right\}=s^{\alpha} L\{f(t)\} .
$$




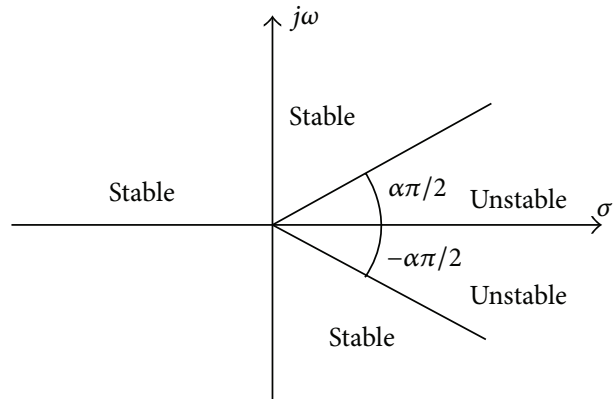

FIGURE 1: The stability region of fractional-order systems.

Another alternative definition of Riemann-Liouville definition of fractional-order derivative was reported by Caputo as follows:

$$
\frac{d^{\alpha} f(t)}{d t^{\alpha}}=\frac{1}{\Gamma(n-\alpha)} \int_{0}^{t} \frac{f^{(n)}(\tau)}{(t-\tau)^{\alpha-n+1}} d \tau .
$$

Since the Caputo definition is more convenient for initial conditions problems, in this paper, the operator $D^{\alpha}$ denotes the $\alpha$-order Caputo fractional derivative.

Then some stability theorems for fractional-order systems are introduced.

Consider the following autonomous system:

$$
\frac{d x}{d t}=A(x) x
$$

One can get the fractional-order form of system (13) as

$$
\frac{d^{\alpha} x}{d t^{\alpha}}=A(x) x
$$

where $x\left(x_{1}, x_{2}, \ldots\right) \in \mathbb{R}^{n}(n \in N), 0<\alpha<1$.

Lemma 3 (see [40]). For fractional-order system (14), we have following.

(i) The system is asymptotically stable if and only if $\left|\arg \left\{\lambda_{i}[A(x)]\right\}\right|>\alpha \pi / 2,(i=1,2, \ldots, n)$, where $\arg \lambda_{i}[A(x)]$ denotes the argument of the matrix $A(x)$ 's eigenvalue $\lambda_{i}$. In this case, components of the state decay toward 0 like $t^{-\alpha}$.

(ii) The system is stable if and only if either it is asymptotically stable or those critical eigenvalues which satisfy $\left|\arg \left(\lambda_{i}(A)\right)\right|=\alpha \pi / 2$ have geometric multiplicity one.

The stability region for $0<a<1$ is illustrated in Figure 1 .

Lemma 4 (see [41]). If system (13) is asymptotically stable, in the range of state variable $x$ (except the origin), all the real part of matrix $A(x)$ 's eigenvalues $\lambda_{i}$ are not more than zero.

Theorem 5. If system (13) is asymptotically stable, fractionalorder system (14) is also asymptotically stable.
Proof. According to Lemma 4, assume that system (13) is asymptotically stable, one can get that all the real part of the matrix $A(x)$ 's eigenvalues $\lambda_{i}$ are not more than zero. Then one can obtain $|\arg (\lambda)|>\pi / 2$. It satisfies $|\arg (\lambda)| \geq a \pi / 2$ for $0<\alpha<1$. According to Lemma 3, fractional-order system (14) is stable.

\section{Adaptive Inverse Optimal Control of a Novel Fractional-Order Four-Wing Hyperchaotic System with Uncertain Parameter}

3.1. Analysis of a Novel Pseudo Four-Wing Hyperchaotic System. We consider a hyperchaotic system described by

$$
\begin{gathered}
\frac{d x_{1}}{d t}=a\left(-x_{1}+x_{2} x_{3}\right), \\
\frac{d x_{2}}{d t}=b x_{2}-h x_{1} x_{3}+c x_{4}, \\
\frac{d x_{3}}{d t}=k x_{1} x_{2}-g x_{3}, \\
\frac{d x_{4}}{d t}=-\left(m x_{2}+n x_{3}\right),
\end{gathered}
$$

where $x_{1}, x_{2}, x_{3}$, and $x_{4}$ are state variables and $a, b, c, h, k, g$, $m$, and $n$ are positive real constants.

Jacobian matrix is equal to

$$
J=\left[\begin{array}{cccc}
-a & a x_{3} & a x_{2} & 0 \\
-h x_{3} & b & -h x_{1} & c \\
k x_{2} & k x_{1} & -g & 0 \\
0 & -m & -n & 0
\end{array}\right]
$$

With parameters $a=10, b=2.5, c=1, h=1, k=4, g=4$, $m=1$, and $n=1 / 8$, system (15) has three equilibrium points: $P_{1}(0,0,0,0), P_{2}(-8,1,-8,61.5)$, and $P_{3}(-8,-1,8,-61.5)$.

Then let $|J-\lambda I|=0$; eigenvalues of matrix $J$ for equilibrium point $P_{1}$ are $\lambda_{1}^{\left(p_{1}\right)}=0.5, \lambda_{2}^{\left(p_{1}\right)}=2, \lambda_{3}^{\left(p_{1}\right)}=$ -10 , and $\lambda_{4}^{\left(p_{1}\right)}=-4$, where $\lambda_{1}^{\left(p_{1}\right)}$ and $\lambda_{2}^{\left(p_{1}\right)}$ are positive roots and $\lambda_{3}^{\left(p_{1}\right)}$ and $\lambda_{4}^{\left(p_{1}\right)}$ are negative roots. For equilibrium points $P_{2}$ and $P_{3}$, eigenvalues of matrix $J$ are $\lambda_{1}^{\left(p_{2,3}\right)}=-11.8430$, $\lambda_{2}^{\left(p_{2,3}\right)}=0.007799, \lambda_{3}^{\left(p_{2,3}\right)}=0.1676-29.4284 i$, and $\lambda_{4}^{\left(p_{2,3}\right)}=$ $0.1676+29.4284 i$, where $\lambda_{1}^{\left(p_{2,3}\right)}$ is a negative root, $\lambda_{2}^{\left(p_{2,3}\right)}$ is a positive root, and $\lambda_{3}^{\left(p_{2,3}\right)}$ and $\lambda_{4}^{\left(p_{2,3}\right)}$ are a pair of conjugate complex roots with positive real part. Therefore, equilibrium points $P_{1}, P_{2}$, and $P_{3}$ are all unstable.

The divergence is given by

$$
\nabla V=\frac{\partial \dot{x}}{\partial x}+\frac{\partial \dot{y}}{\partial y}+\frac{\partial \dot{z}}{\partial z}+\frac{\partial \dot{v}}{\partial v}=-11.5<0 .
$$

System (15) is dissipative.

Figure 2 shows Lyapunov exponents versus $b$. When $b=$ 2.5, Lyapunov exponents are as follows: $\lambda_{L 1}=0.602, \lambda_{L 2}=$ 


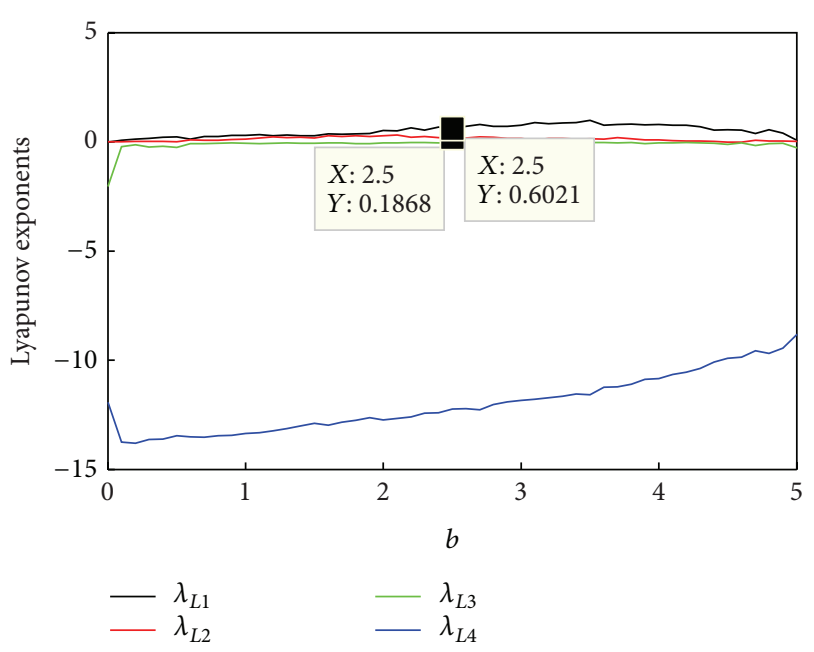

FIGURE 2: Lyapunov exponents versus $b$.

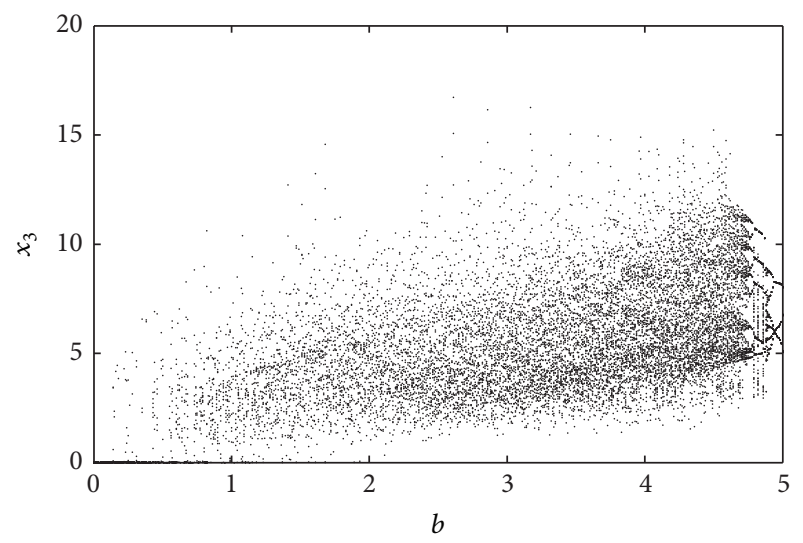

FIGURE 3: Bifurcation diagram of $x_{3}$ versus $b$.

0.187, $\lambda_{L 3}=-0.173, \lambda_{L 4}=-12.166 . \lambda_{L 1}, \lambda_{L 2}>0$, and $\lambda_{L 3}, \lambda_{L 4}<0$, so system (15) is in hyperchaotic state.

Figure 3 is the bifurcation diagram of $x_{3}$ versus $b$. The image shows complex and rich dynamical behaviors.

In the process of investigating this hyperchaotic system, we found this odd: different initial values would generate different area of attractor. With the aforementioned set of parameters and the initial values $(0.1,0.4,0.9,1)$, $(0.1,0.4,-0.9,1)$, one can get the strange attractor as shown in Figure 4, where the upper one is indicated by a solid line and the lower one is indicated by a dotted line.

To explain this weird phenomenon, detailed mathematical deduction and simulations will be given.

Firstly, observing the first and third equations in system (15), one has

$$
\begin{aligned}
& \frac{d x_{1}}{d t}=a\left(-x_{1}+x_{2} x_{3}\right) \\
& \frac{d x_{3}}{d t}=k x_{1} x_{2}-g x_{3} .
\end{aligned}
$$

Multiplying (18) with $x_{1}$ and (19) with $x_{3}$ yields

$$
\begin{aligned}
& \frac{d x_{1}}{d t} x_{1}=-a x_{1}^{2}+a x_{1} x_{2} x_{3} \\
& \frac{d x_{3}}{d t} x_{3}=k x_{1} x_{2} x_{3}-g x_{3}^{2} .
\end{aligned}
$$

Equation (20) can be rewritten as

$$
\begin{aligned}
& \frac{1}{a} \frac{d x_{1}}{d t} x_{1}=-x_{1}^{2}+x_{1} x_{2} x_{3} \\
& \frac{1}{k} \frac{d x_{3}}{d t} x_{3}=x_{1} x_{2} x_{3}-\frac{g}{k} x_{3}^{2}
\end{aligned}
$$

Subtracting (22) from (21), one obtains

$$
\frac{1}{k} \frac{d x_{3}}{d t} x_{3}-\frac{1}{a} \frac{d x_{1}}{d t} x_{1}=x_{1}^{2}-\frac{g}{k} x_{3}^{2} .
$$

Equation (23) is equivalent to

$$
\frac{d\left(a x_{3}^{2}-k x_{1}^{2}\right)}{d t}=2\left(a k x_{1}^{2}-a g x_{3}^{2}\right)
$$

Equation (24) can be rewritten as

$$
\frac{d\left[\left(\sqrt{a} x_{3}\right)^{2}-\left(\sqrt{k} x_{1}\right)^{2}\right]}{d t}=2\left[a\left(\sqrt{k} x_{1}\right)^{2}-g\left(\sqrt{a} x_{3}\right)^{2}\right] .
$$

By assumption, $a>g>0$, let $a=\mu+g$, where $\mu$ is a positive real constant and $\mu>0$. Equation (25) is equivalent to

$$
\begin{aligned}
& \frac{d\left[\left(\sqrt{a} x_{3}\right)^{2}-\left(\sqrt{k} x_{1}\right)^{2}\right]}{d t} \\
& \quad=-2 g\left[\left(\sqrt{a} x_{3}\right)^{2}-\left(\sqrt{k} x_{1}\right)^{2}\right]+2 \mu\left(\sqrt{a} x_{1}\right)^{2} .
\end{aligned}
$$

Solving (26) yields

$$
\begin{gathered}
\left(\sqrt{a} x_{3}(t)\right)^{2}-\left(\sqrt{k} x_{1}(\mathrm{t})\right)^{2} \\
=e^{-2 g t}\left[\int_{0}^{t} 2 \mu\left(\sqrt{a} x_{1}(\mathrm{t})\right)^{2} e^{2 g \tau} d \tau+\left(\sqrt{a} x_{3}(0)\right)^{2}\right. \\
\left.-\left(\sqrt{k} x_{1}(0)\right)^{2}\right]
\end{gathered}
$$

Since $e^{-2 g t}>0,2 \mu\left(\sqrt{a} x_{1}(t)\right)^{2} e^{2 g \tau}>0$; if the initial values of system (15) at time satisfy $\left|\sqrt{a} x_{3}(0)\right|>\left|\sqrt{k} x_{1}(0)\right|$ or $\left(\sqrt{a} x_{3}(0)\right)^{2}>\left(\sqrt{k} x_{1}(0)\right)^{2}$, then (26) implies that $\left(\sqrt{a} x_{3}(t)\right)^{2}-$ $\left(\sqrt{k} x_{1}(t)\right)^{2}>0$ for all $t \geq 0$. 


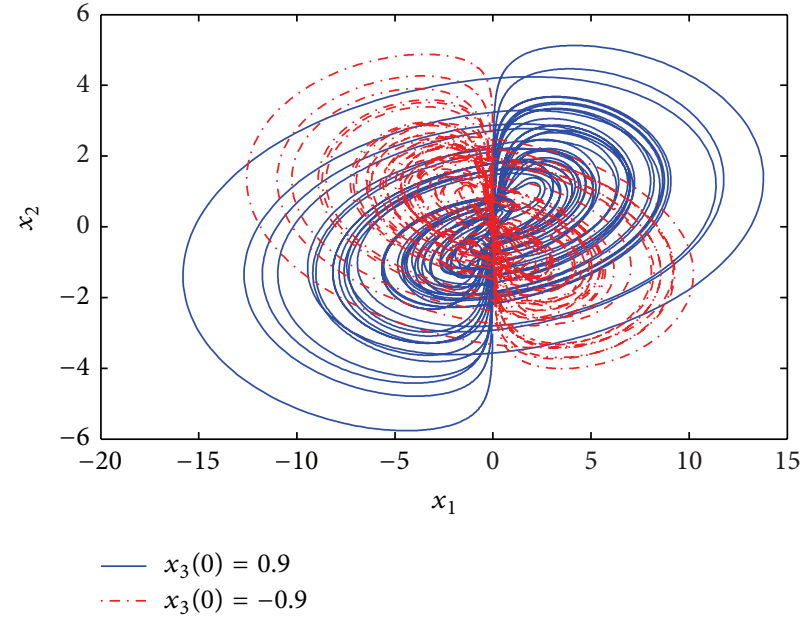

(a) Projection on the $x_{1}-x_{2}$ plane

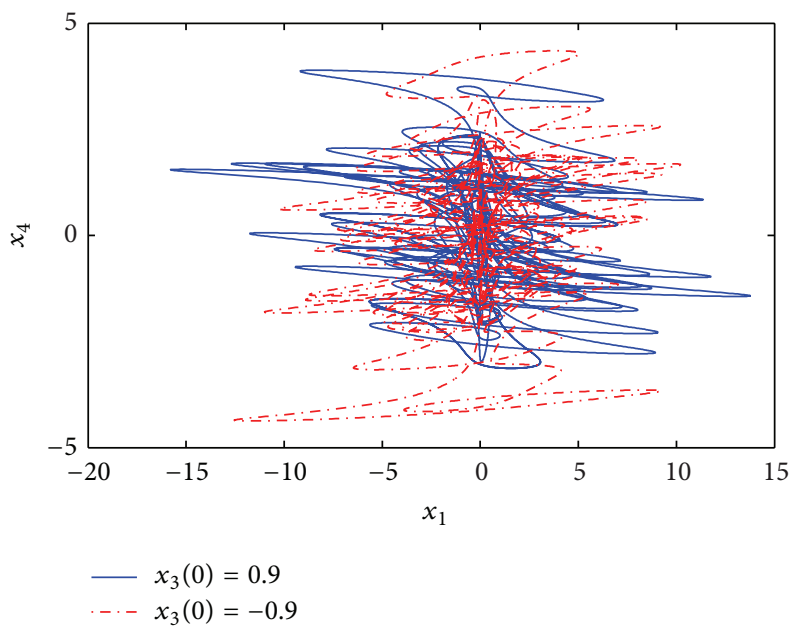

(c) Projection on the $x_{1}-x_{4}$ plane

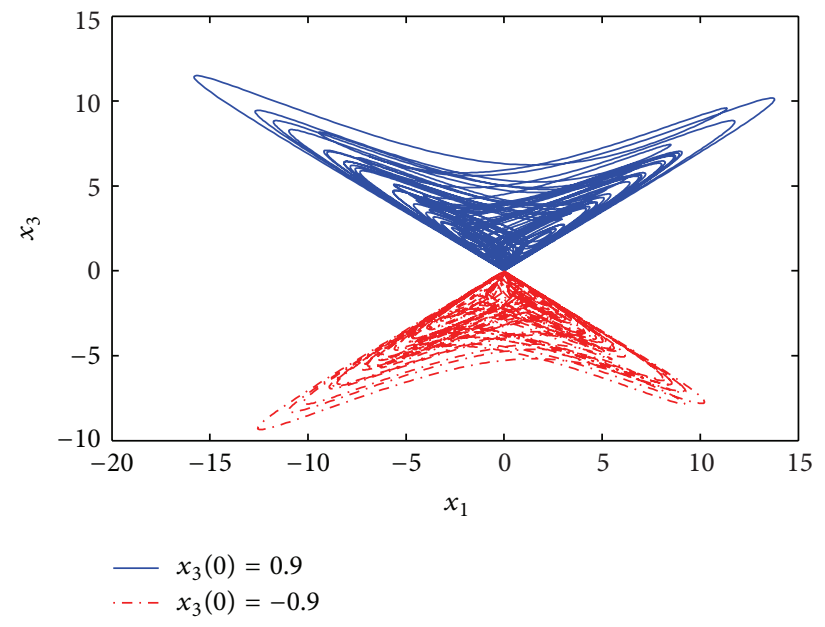

(b) Projection on the $x_{1}-x_{3}$ plane

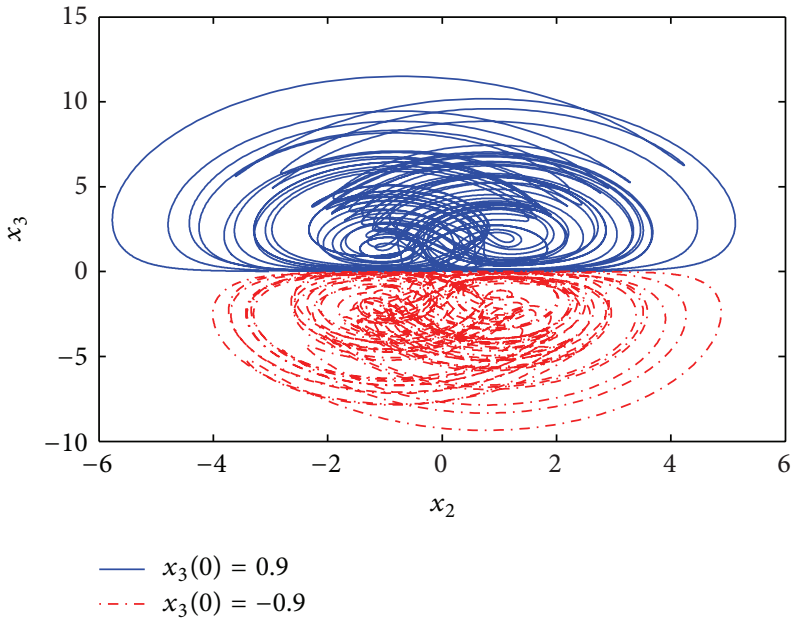

(d) Projection on the $x_{2}-x_{3}$ plane

FIGURE 4: Chaotic attractor of system (15).

State variable $x_{3}(t)$ cannot cross the plane $x_{3}=0$ (Figure 10). The trajectories of system (15) are limited in the area of $\left|\sqrt{a} x_{3}(t)\right|>\left|\sqrt{k} x_{1}(t)\right|$. If the trajectories of system (15) travel from the upper part to the lower part, there will be $t_{1}>0$ at $x_{3}\left(t_{1}\right)=0$. That contradicts the fact that $\left(\sqrt{a} x_{3}(t)\right)^{2}>\left(\sqrt{k} x_{1}(t)\right)^{2}$ for all $t \geq 0$.

For example, with the aforementioned set of parameters and $a>g>0$, the initial values are $(0.1,0.4,0.9,1)$ and $(0.1,0.4,-0.9,1)$, respectively, which all belong to the area of $\left|\sqrt{a} x_{3}(0)\right|>\left|\sqrt{k} x_{1}(0)\right|$, and one can find two coexisting attractors: the upper one with the initial value $(0.1,0.4,0.9,1)$ and the lower one with the initial value $(0.1,0.4,-0.9,1)$, as shown in Figure 5. The trajectories of the attractor can travel only within the area $\left|\sqrt{a} x_{3}(t)\right|>\left|\sqrt{k} x_{1}(t)\right|$ limited by the dashed line and cannot cross the plane $x_{3}=0$. That means that system (15) can only generate pseudo four-wing attractor.
To produce a real four-wing attractor, we introduce a simple linear state feedback $e x_{4}$ to the third equation of system (15). Then one gets the following system:

$$
\begin{gathered}
\frac{d x_{1}}{d t}=a\left(-x_{1}+x_{2} x_{3}\right), \\
\frac{d x_{2}}{d t}=b x_{2}-h x_{1} x_{3}+c x_{4}, \\
\frac{d x_{3}}{d t}=k x_{1} x_{2}-g x_{3}+e x_{4}, \\
\frac{d x_{4}}{d t}=-\left(m x_{2}+n x_{3}\right) .
\end{gathered}
$$

With the aforementioned set of parameters and $e=1$, the real four-wing attractor is shown in Figure 6. 


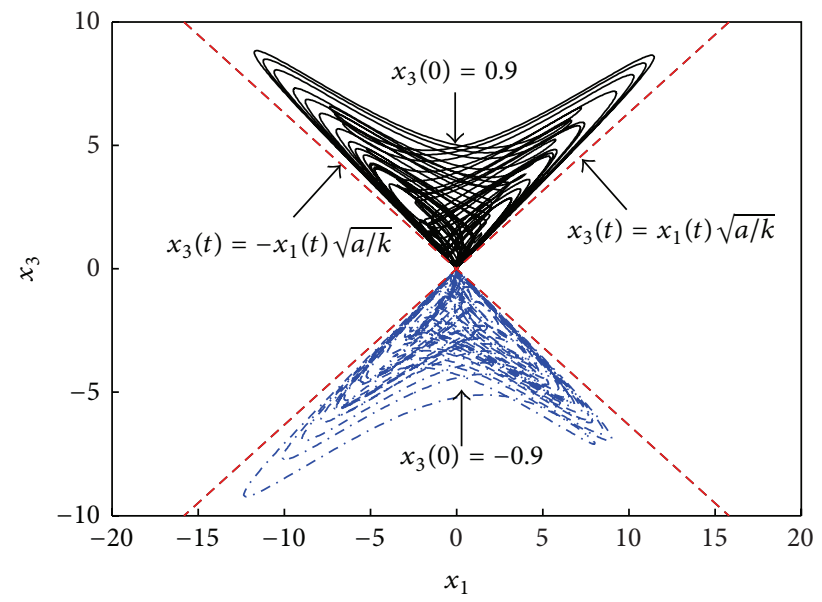

FIGURE 5: Two coexisting attractors of $x_{1}-x_{3}$ phase plane.

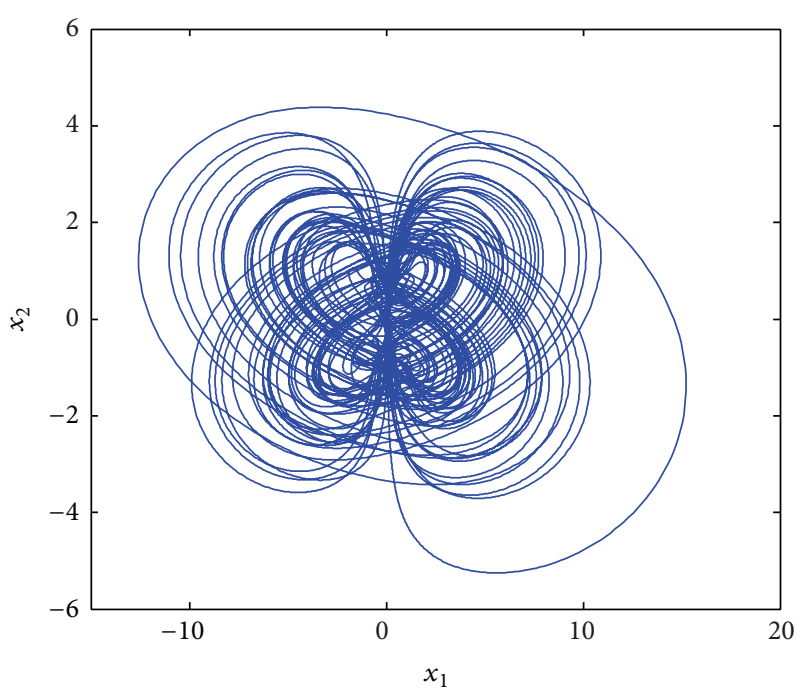

(a) Projection on the $x_{1}-x_{2}$ plane

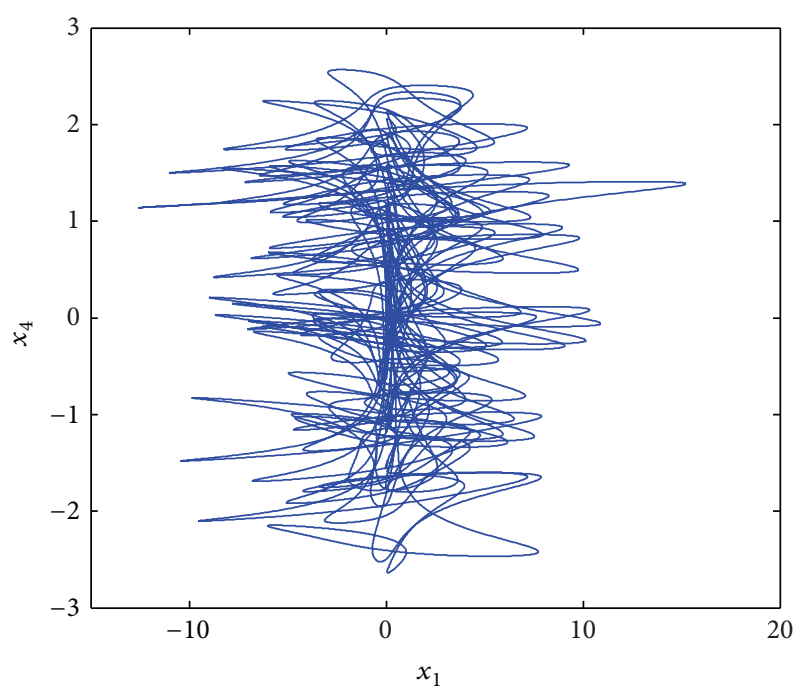

(c) Projection on the $x_{1}-x_{4}$ plane

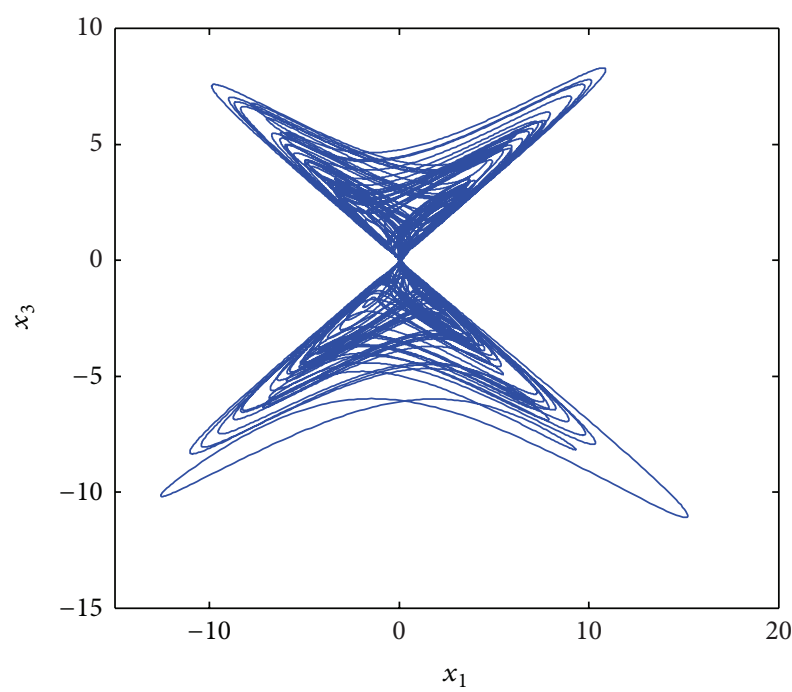

(b) Projection on the $x_{1}-x_{3}$ plane

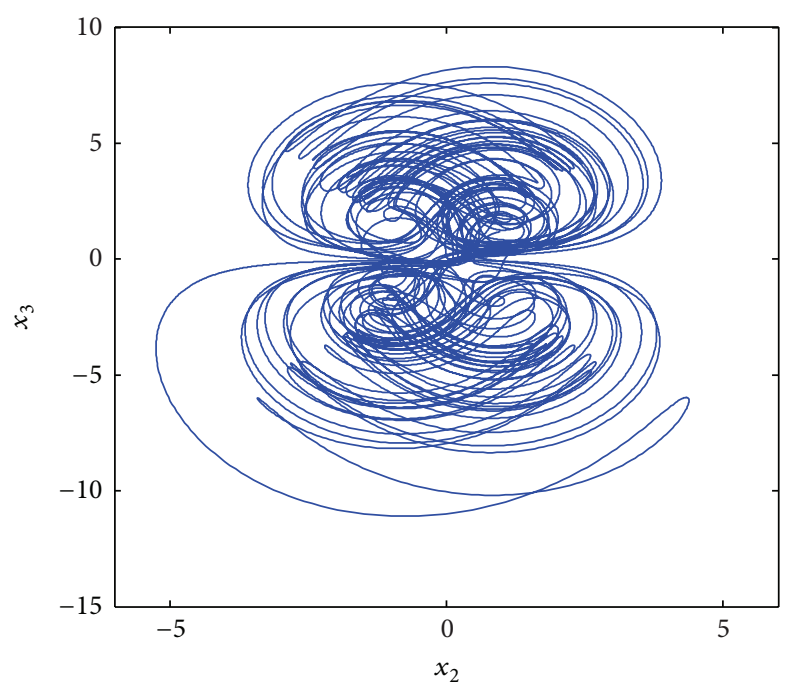

(d) Projection on the $x_{2}-x_{3}$ plane

Figure 6: The four-wing attractor of system (28). 


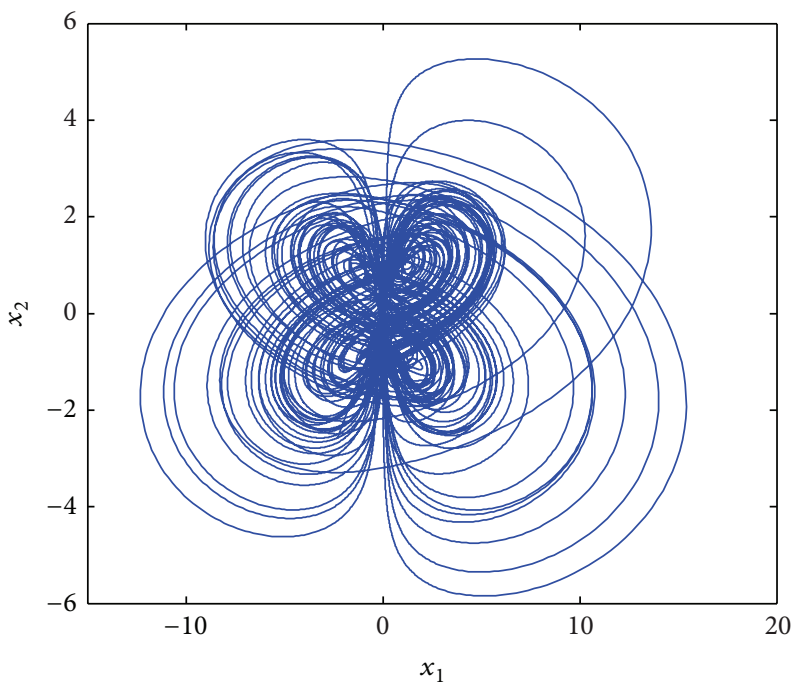

(a) Projection on the $x_{1}-x_{2}$ plane

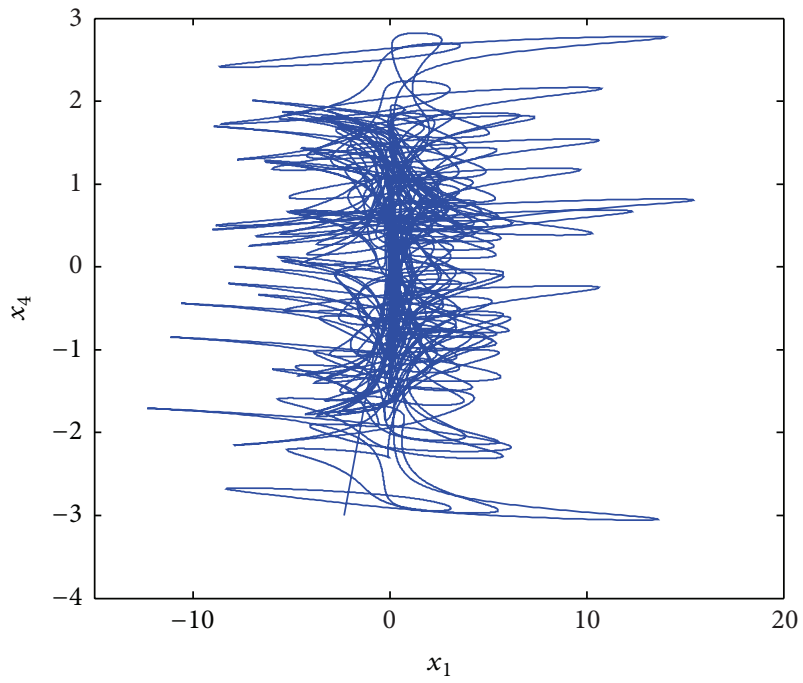

(c) Projection on the $x_{1}-x_{4}$ plane

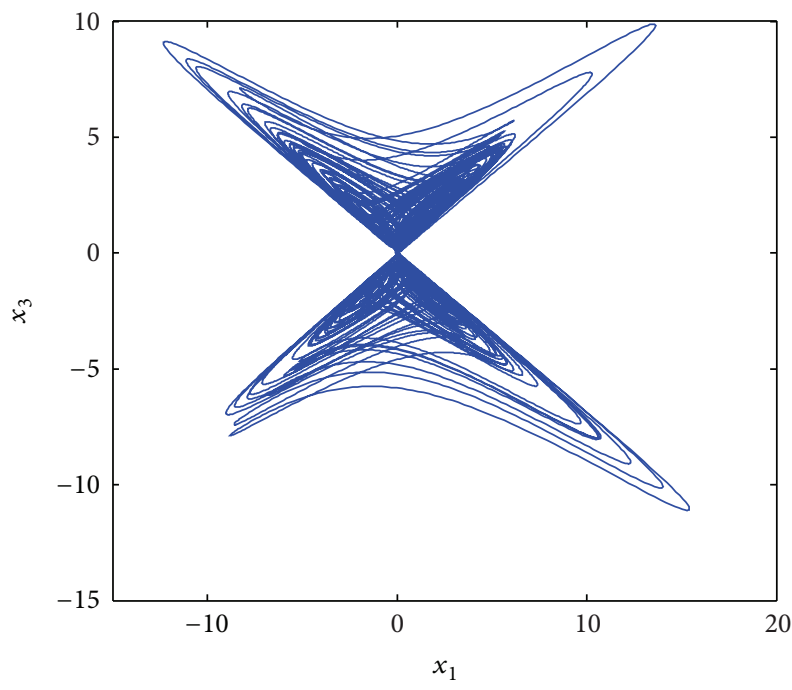

(b) Projection on the $x_{1}-x_{3}$ plane

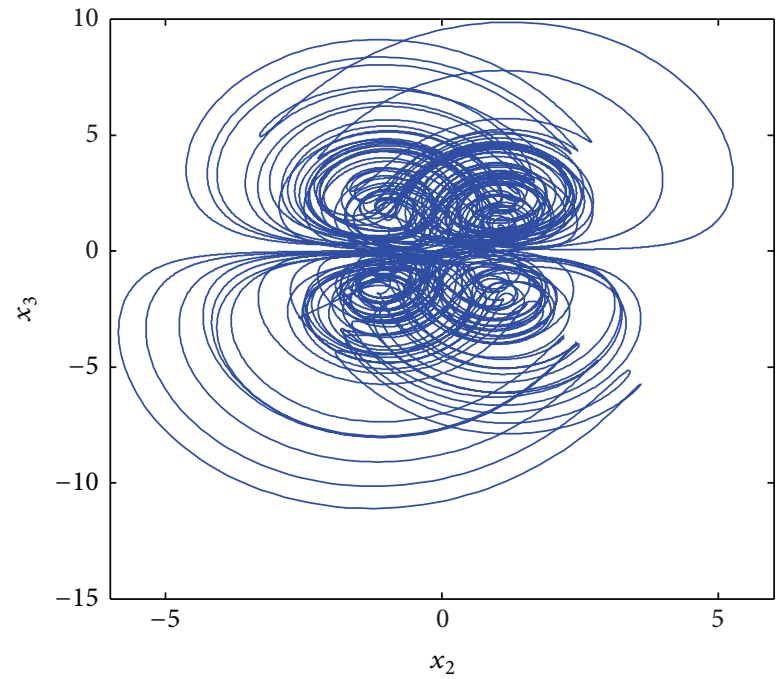

(d) Projection on the $x_{2}-x_{3}$ plane

FiguRE 7: Fractional-order four-wing attractor of system (29) for $\alpha=0.9$.

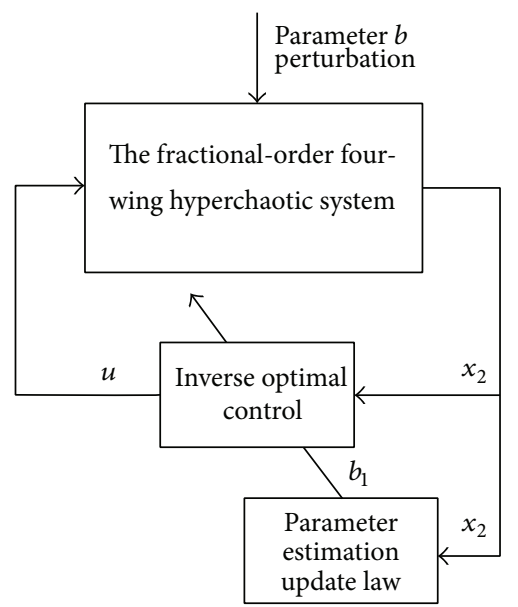

FIGURE 8: Blocks diagram for adaptive inverse optimal control. 
3.2. Adaptive Inverse Optimal Control of the Fractional-Order Four-Wing Hyperchaotic System with Uncertain Parameter. The fractional-order hyperchaotic system with four-wing attractor can be constructed as the following form:

$$
\begin{gathered}
\frac{d^{\alpha} x_{1}}{d t^{\alpha}}=a\left(-x_{1}+x_{2} x_{3}\right), \\
\frac{d^{\alpha} x_{2}}{d t^{\alpha}}=b x_{2}-h x_{1} x_{3}+c x_{4}, \\
\frac{d^{\alpha} x_{3}}{d t^{\alpha}}=k x_{1} x_{2}-g x_{3}+e x_{4}, \\
\frac{d^{\alpha} x_{4}}{d t^{\alpha}}=-\left(m x_{2}+n x_{3}\right) .
\end{gathered}
$$

The predictor-corrector method is used to implement the fractional-order four-wing hyperchaotic system with the order $\alpha=0.9$. The fractional-order four-wing attractor of system (29) with the aforementioned set of parameters is shown in Figure 7.

Then the inverse optimal control methodology is developed to achieve the global asymptotic stabilization of the fractional-order four-wing hyperchaotic system with uncertain parameter. The closed-loop system with a controller $u$ is described by

$$
\begin{gathered}
\frac{d^{\alpha} x_{1}}{d t^{\alpha}}=a\left(-x_{1}+x_{2} x_{3}\right), \\
\frac{d^{\alpha} x_{2}}{d t^{\alpha}}=b(t) x_{2}-h x_{1} x_{3}+c x_{4}+u, \\
\frac{d^{\alpha} x_{3}}{d t^{\alpha}}=k x_{1} x_{2}-g x_{3}+e x_{4}, \\
\frac{d^{\alpha} x_{4}}{d t^{\alpha}}=-\left(m x_{2}+n x_{3}\right) .
\end{gathered}
$$

With the aforementioned set of parameters, $b(t)$ is an time-varying uncertain parameter described by $b(t)=b+$ $\Delta b(t), b$ is positive definite, and $\Delta b(t)$ is a bounded function which satisfied $|\Delta b(t)| \leq \xi$.

Theorem 6. The fractional-order hyperchaotic system with four-wing attractor can achieve global asymptotical stability by the following linear state feedback control law:

$$
u=-\left(b_{1}+\lambda\right) x_{2}
$$

where $b_{1}$ is the estimate value of the unknown parameter $b$ and $\lambda>0$. The parameter estimation update law $\dot{b}_{1}$ is

$$
\dot{b}_{1}=\frac{4 a x_{2}^{2}}{h}
$$

Proof. According to Theorem 5, one considers the integerorder dynamical system as follows:

$$
\begin{gathered}
\frac{d x_{1}}{d t}=a\left(-x_{1}+x_{2} x_{3}\right) \\
\frac{d x_{2}}{d t}=b(t) x_{2}-h x_{1} x_{3}+c x_{4}+u \\
\frac{d x_{3}}{d t}=k x_{1} x_{2}-g x_{3}+e x_{4} \\
\frac{d x_{4}}{d t}=-\left(m x_{2}+n x_{3}\right) .
\end{gathered}
$$

Construct a Lyapunov function for system (33). Consider

$$
V=x_{1}^{2}+\frac{2 a}{h} x_{2}^{2}+\frac{a}{k} x_{3}^{2}+2 a x_{4}^{2}+\frac{1}{2} \widetilde{b}^{2},
$$

where $a>0, c>0, h>0, k>0$, and $\tilde{b}=b-b_{1}$. Obviously, $V(x)$ is positive definite. The derivative of $V(x)$ along the time is

$$
\dot{V}(x)=2 x_{1} \dot{x}_{1}+\frac{4 a x_{2}}{h} \dot{x}_{2}+\frac{2 a x_{3}}{k} \dot{x}_{3}+4 a x_{4} \dot{x}_{4}-\tilde{b} \dot{b}_{1} .
$$

Substituting (32) and (33) into (35) yields

$$
\begin{aligned}
\dot{V}(x)= & 2 a\left(-x_{1}+x_{2} x_{3}\right) x_{1} \\
& +\frac{4 a}{h}\left(b(t) x_{2}-h x_{1} x_{3}+c x_{4}+u\right) x_{2} \\
& +\frac{2 a}{k}\left(k x_{1} x_{2}-g x_{3}+e x_{4}\right) x_{3} \\
& -4 a\left(m x_{2}+n x_{3}\right) x_{4}-\left(b-b_{1}\right) \frac{4 a x_{2}^{2}}{h} .
\end{aligned}
$$

Equation (36) can be rewritten as

$$
\dot{V}(x)=-2 a x_{1}^{2}+\frac{4 a\left(b_{1}+\Delta b(t)\right)}{h} x_{2}^{2}-\frac{2 a g}{k} x_{3}^{2}+\frac{4 a}{h} u x_{2} .
$$

According to Definition 1, one can obtain

$$
\begin{gathered}
L_{f} V=-2 a x_{1}^{2}+\frac{4 a\left(b_{1}+\Delta b(t)\right)}{h} x_{2}^{2}-\frac{2 a g}{k} x_{3}^{2}, \\
L_{g} V=\frac{4 a}{h} x_{2} .
\end{gathered}
$$

It is easy to verify that $L_{f} V<0$ for $L_{g} V=0$. Then $V(x)$ is indeed a control Lyapunov function (CLF).

Define a state feedback controller according to the results of Lemma 2:

$$
u=-\beta R(x)^{-1}\left(L_{g} V\right)=-\left(b_{1}+\lambda\right) x_{2}
$$

where $\beta$ is a positive constant and $R(x)^{-1}$ is a positive definite function of $x$. Consider

$$
R(x)^{-1}=\frac{h}{4 a \beta}\left(b_{1}+\lambda\right) .
$$




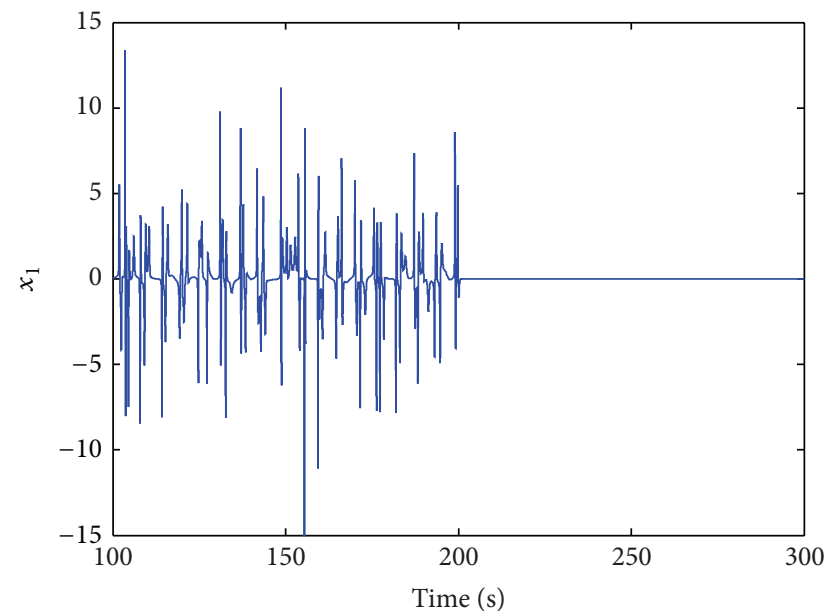

(a) $x_{1}$ state

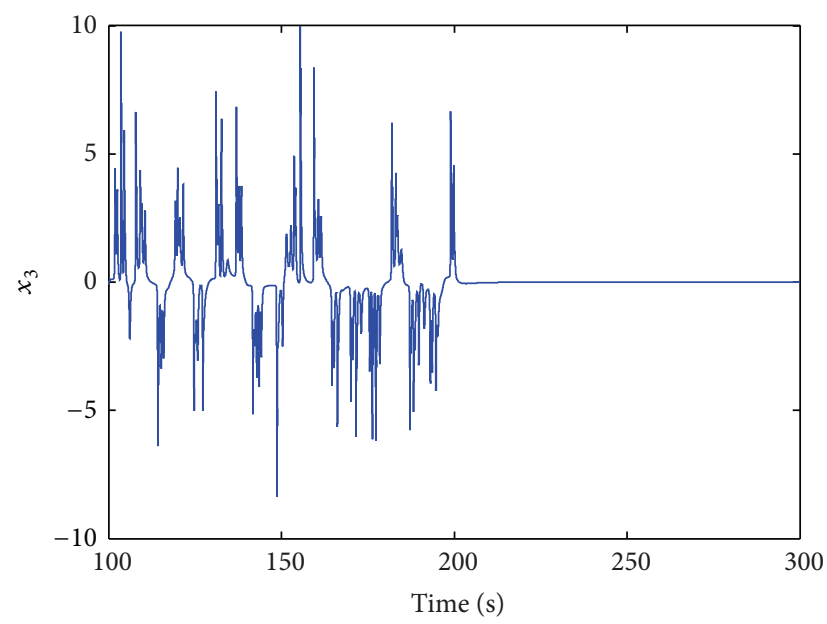

(c) $x_{3}$ state

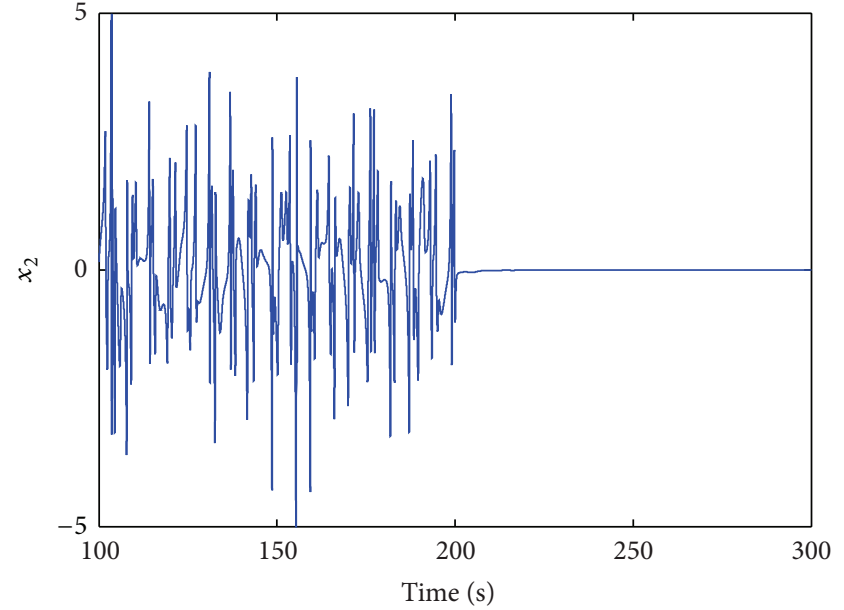

(b) $x_{2}$ state

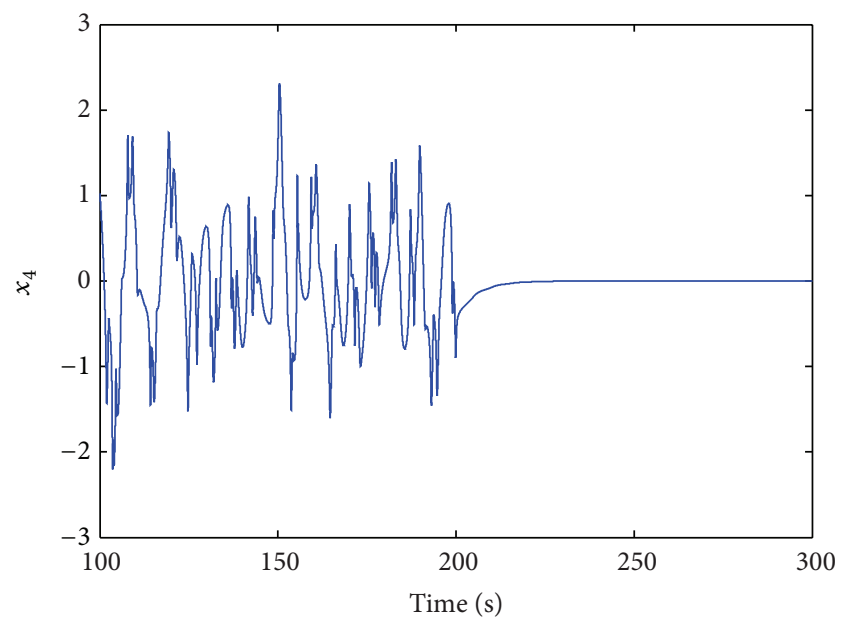

(d) $x_{4}$ state

FIgURE 9: Time waveform of state variables of the controlled fractional-order system with the controller started at $t=200 \mathrm{~s}$.

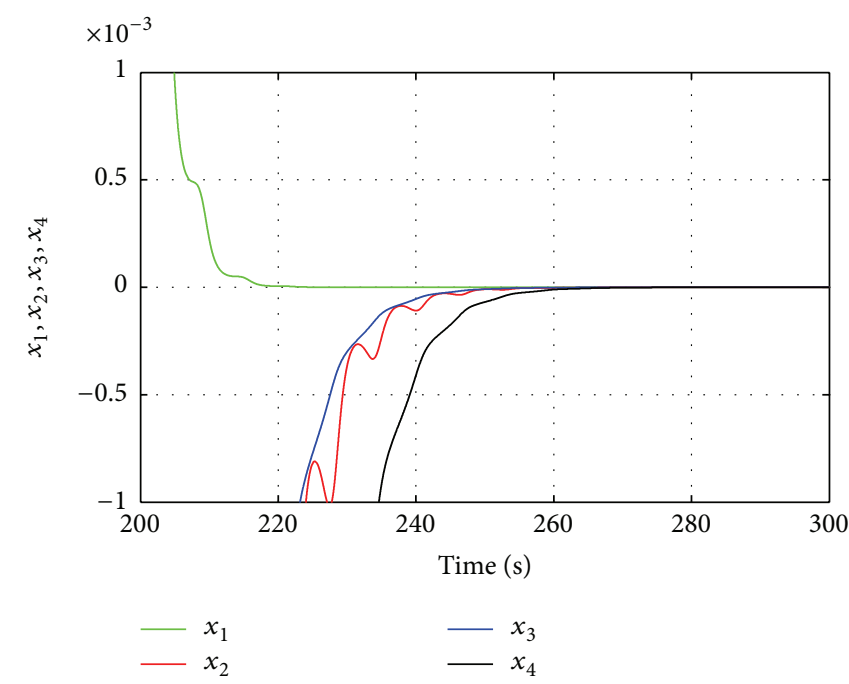

FIGURE 10: The time evolutions of each state variable starting at time $t=200 \mathrm{~s}$. 


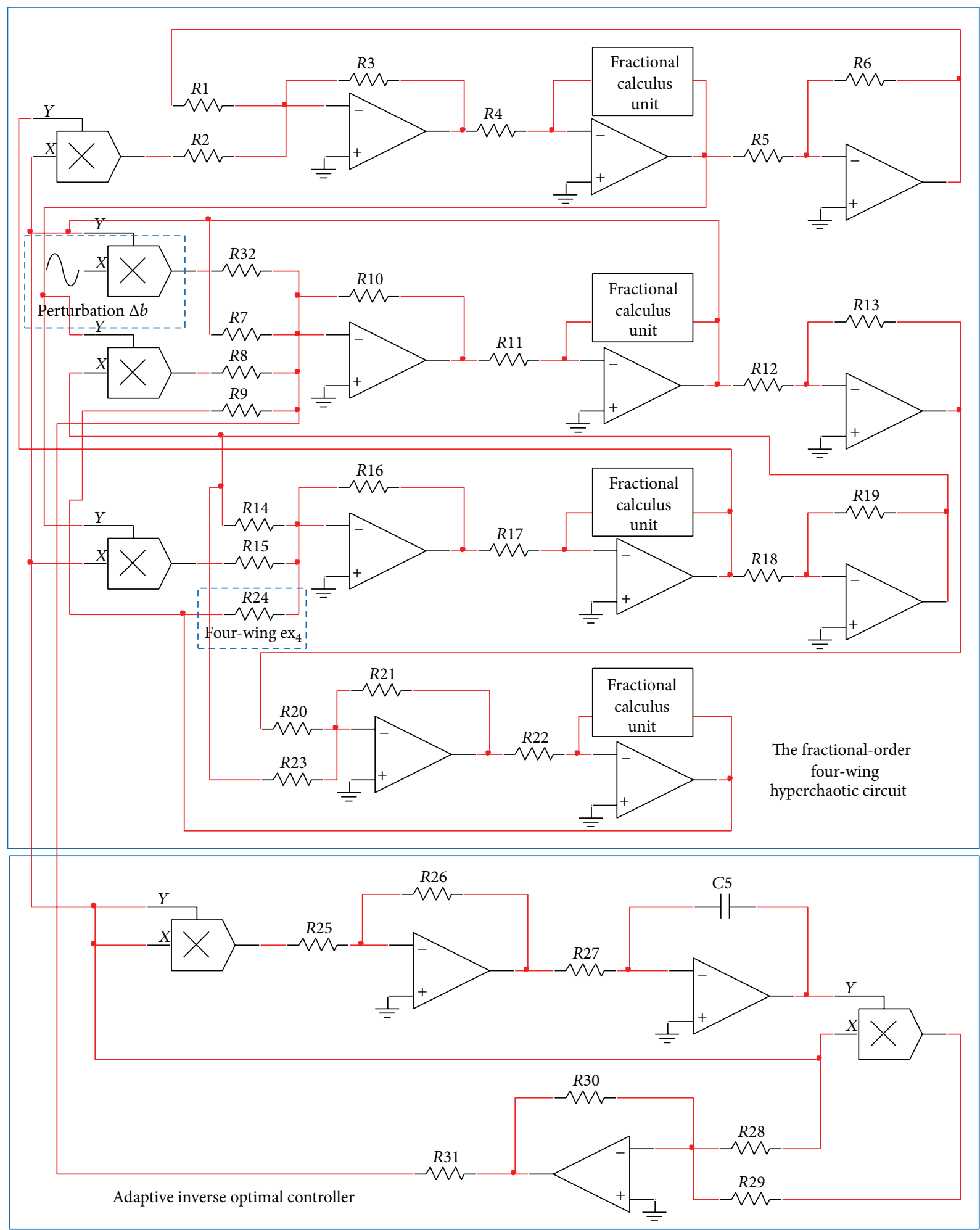

FIGURE 11: Analog electronic circuit implementations of the fractional-order four-wing hyperchaotic system and the adaptive inverse optimal control. 


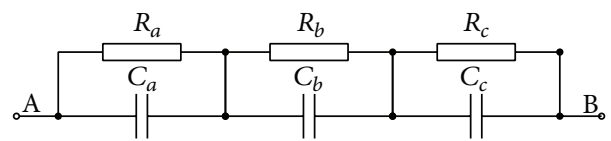

Figure 12: Chain fractance.

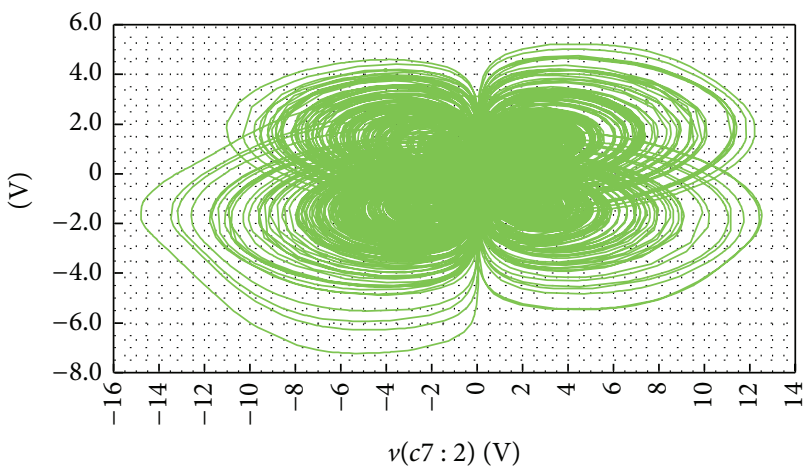

$\square V(U 13: Y 1)$

(a) Projection on the $x_{1}-x_{2}$ plane

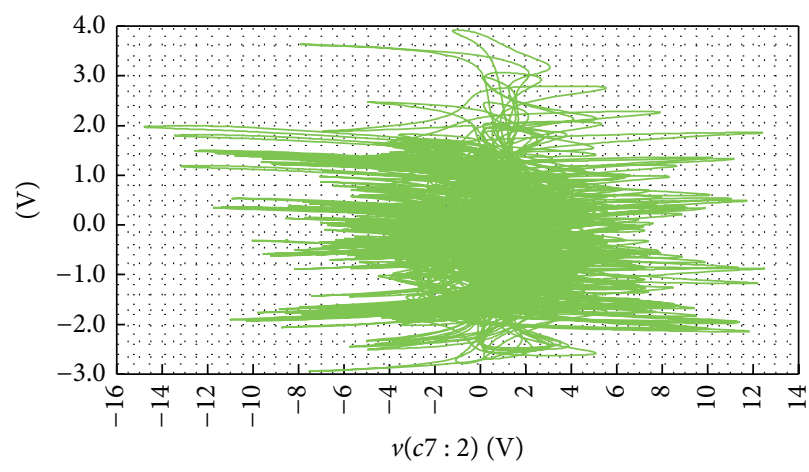

$\square V(C 16: 2)$

(c) Projection on the $x_{1}-x_{4}$ plane

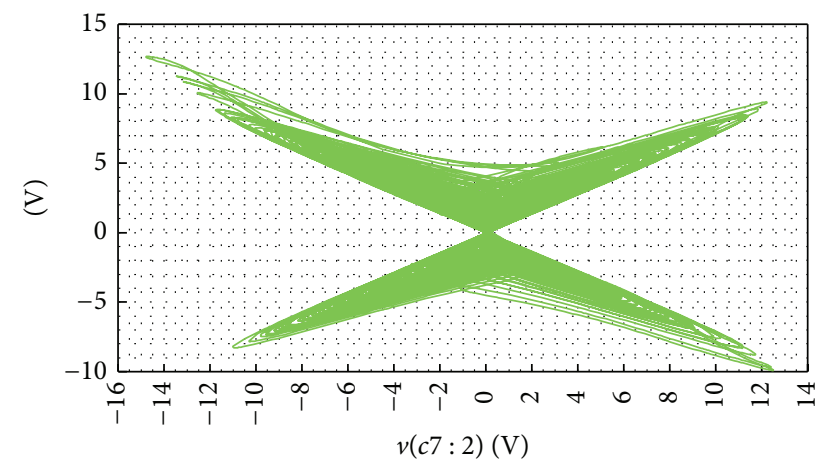

$\square V(U 13: X 1)$

(b) Projection on the $x_{1}-x_{3}$ plane

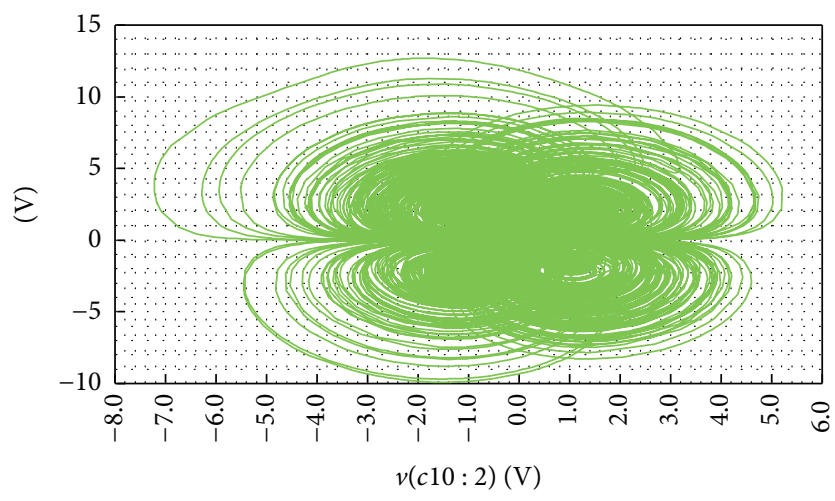

$\square V(U 13: X 1)$

(d) Projection on the $x_{2}-x_{3}$ plane

FIGURE 13: Phase portrait of the real four-wing attractor by Pspice.

Substituting (39) into (37) yields

$$
\dot{V}(x)=-2 a x_{1}^{2}-\frac{4 a(\lambda-\Delta b(t))}{h} x_{2}^{2}-\frac{2 a g}{k} x_{3}^{2} .
$$

According to the definition of $\Delta b(t)$, if $\lambda$ satisfies $\lambda \geq$ $|\Delta b(t)|=\xi$, it can be proved that $\dot{V}(x)$ is negative definite for all $x \neq 0$ easily.

That means the close-loop system can achieve global asymptotical stabilization with the controller.

Define a cost functional as follows:

$$
J(u)=\lim _{t \rightarrow \infty}\left\{2 \beta V(x)+\int_{0}^{t}\left(l(x)+u^{T} R(x) u\right) d \tau\right\},
$$

where

$$
l(x)=-\beta L_{f} V+\beta^{2} R(x)^{-1}\left(L_{g} V\right)^{2} .
$$

Substituting (38) into (43) yields

$$
\begin{aligned}
l(x)= & -\beta\left(-2 a x_{1}^{2}-\frac{2 a g}{k} x_{3}^{2}+\frac{4 a\left(b_{1}+\Delta b(t)\right)}{h} x_{2}^{2}\right) \\
& +\frac{4 a}{h} \beta\left(b_{1}+\lambda\right) x_{2}^{2} .
\end{aligned}
$$

Then

$$
l(x)=2 a \beta x_{1}^{2}+\frac{2 a g \beta}{k} x_{3}^{2}+\frac{4 a \beta}{h}(\lambda-\Delta b(t)) x_{2}^{2} .
$$

Equation (45) implies that $l(x)$ is positive definite for all $x \neq 0$. When $x$ approaches infinity, $l(x)$ also approaches infinity. So function $l(x)$ is radially unbounded.

Substituting (39) into (2) yields

$$
\dot{V}(x)=L_{f} V-\beta R^{-1}(x)\left(L_{g} V\right)^{2} .
$$




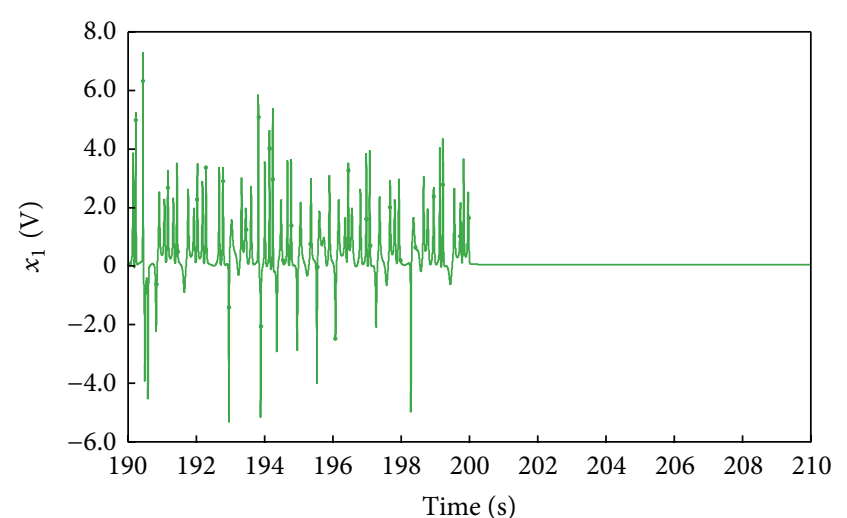

$\square V(U 12: X 1)$

(a) $x_{1}$ state

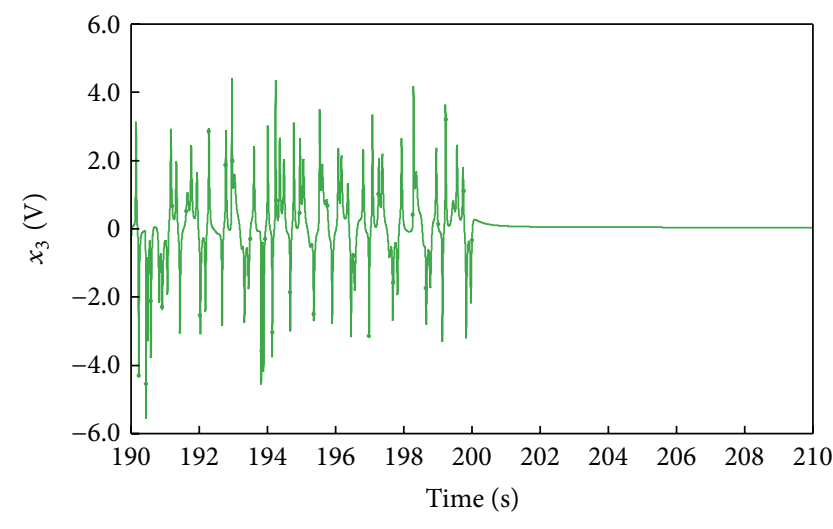

$\square V(U 13: X 1)$

(c) $x_{3}$ state

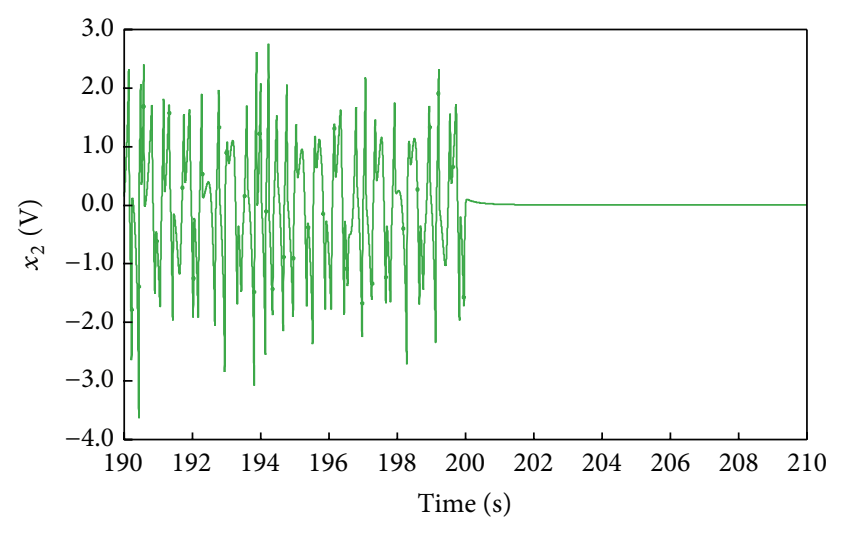

$\square V(U 13: Y 1)$

(b) $x_{2}$ state

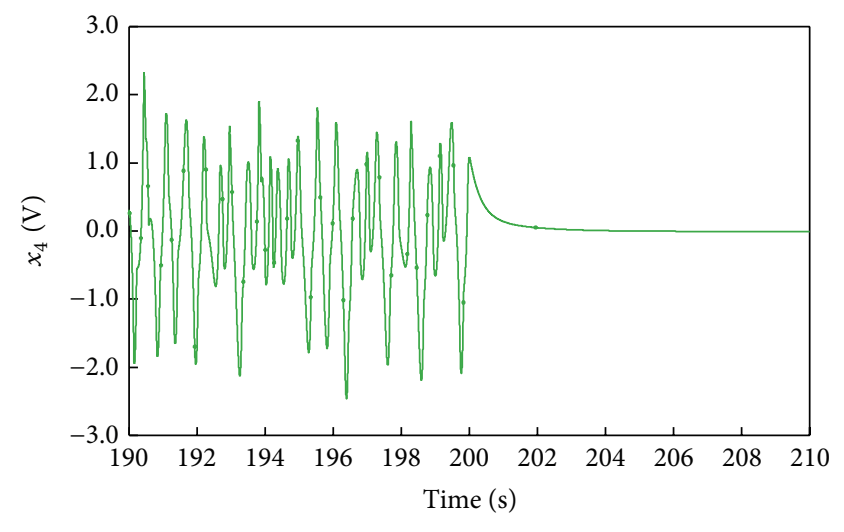

$\square V(C 16: 2)$

(d) $x_{4}$ state

FIGURE 14: Time waveform of the state variables of the controlled fractional-order system with the controller started at $t=200 \mathrm{~s}$ by Pspice.

Multiplying both sides of (46) with $-\beta$, one obtains

$$
-\beta \dot{V}(x)=-\beta L_{f} V+\beta^{2} R(x)^{-1}\left(L_{g} V\right)^{2} .
$$

Substituting (43) into (47) yields

$$
-\beta \dot{V}(x)=l(x) .
$$

According to (39), one obtains

$$
u^{T} R(x) u=\beta^{2} R(x)^{-1}\left(L_{g} V\right)^{2} .
$$

Then

$$
l(x)+u^{T} R(x) u=-\beta \dot{V}(x)+\beta^{2} R(x)^{-1}\left(L_{g} V\right)^{2} .
$$

Substituting (50) into (42) yields

$$
\begin{aligned}
J(u)=\lim _{t \rightarrow \infty}\{ & 2 \beta V(x) \\
& \left.+\int_{0}^{t}\left(-\beta \dot{V}(x)+\beta^{2} R(x)^{-1}\left(L_{g} V\right)^{2}\right) d \tau\right\} .
\end{aligned}
$$

In accordance with the optimal control law, the minimum of the cost function is

$$
J(u)_{\min }=2 \beta V(x(0)) .
$$

Now, the linear state feedback control law (31) has been proved to be the optimal control law. Then integer-order system (33) is stable at zero equilibrium point with the controller $u$. According to Theorem 5, one can obtain that fractional-order four-wing hyperchaotic system (29) is also stable at the zero equilibrium point because all the real parts of matrix $A(x)$ 's eigenvalues are not more than zero.

The blocks diagram for adaptive inverse optimal control of the fractional-order four-wing hyperchaotic system is shown in Figure 8.

The perturbation of parameter $b(t)$ is given by $b(t)=$ $2.5+\sin (7 t)$. The state trajectories of the controlled fractionalorder hyperchaotic system are displayed in Figure 9.

When $t=200 \mathrm{~s}$, the controller begins to work. States converge to zero with the parameter perturbation which indicates the former uncertainty. The fractional-order fourwing hyperchaotic system is indeed globally asymptotically stabilized by the adaptive inverse optimal controller. 


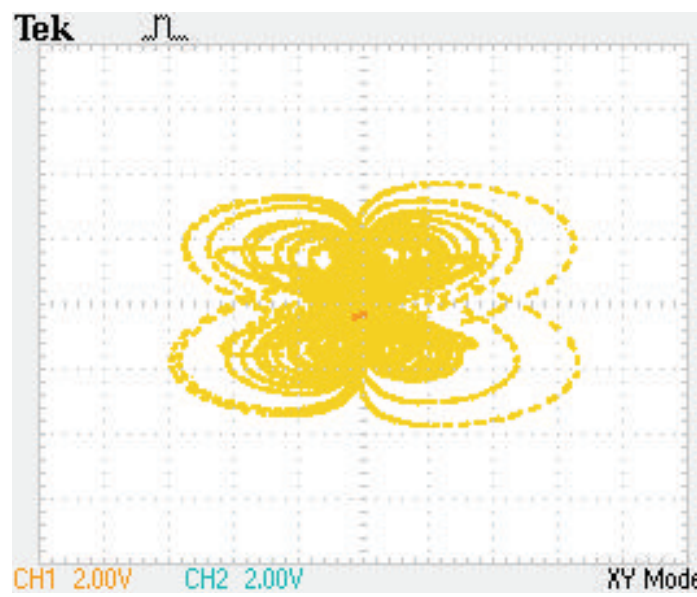

(a) Projection on the $x_{1}-x_{2}$ plane

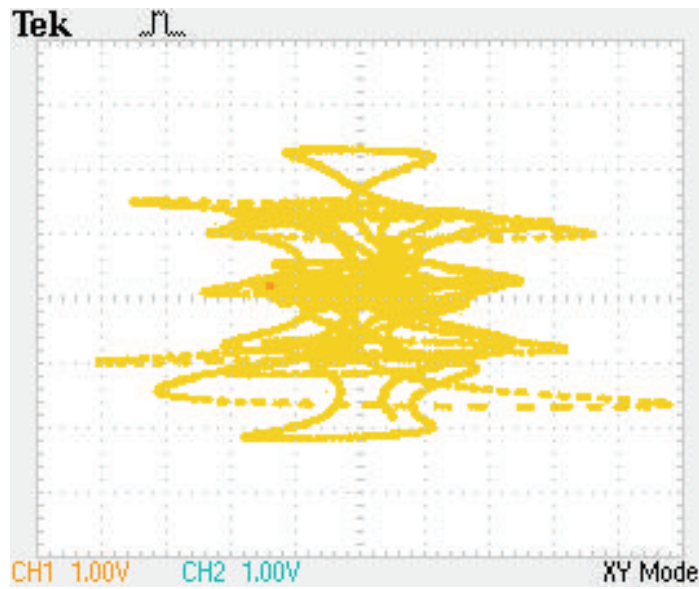

(c) Projection on the $x_{1}-x_{4}$ plane

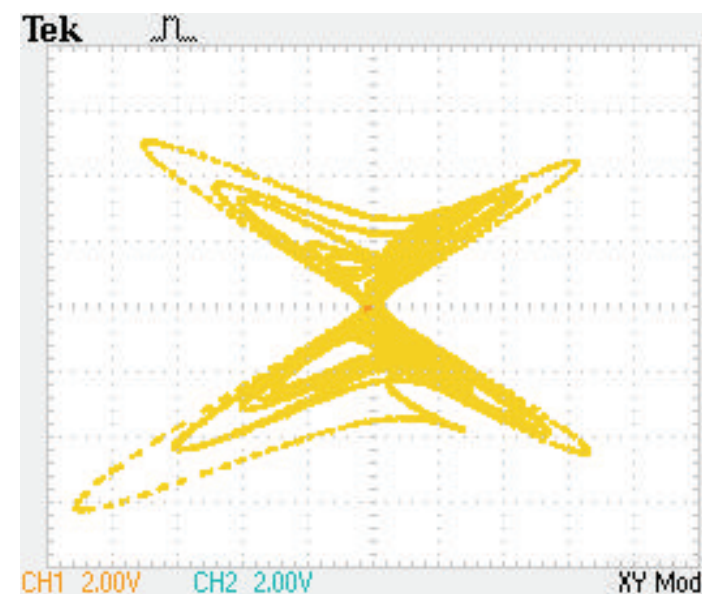

(b) Projection on the $x_{1}-x_{3}$ plane

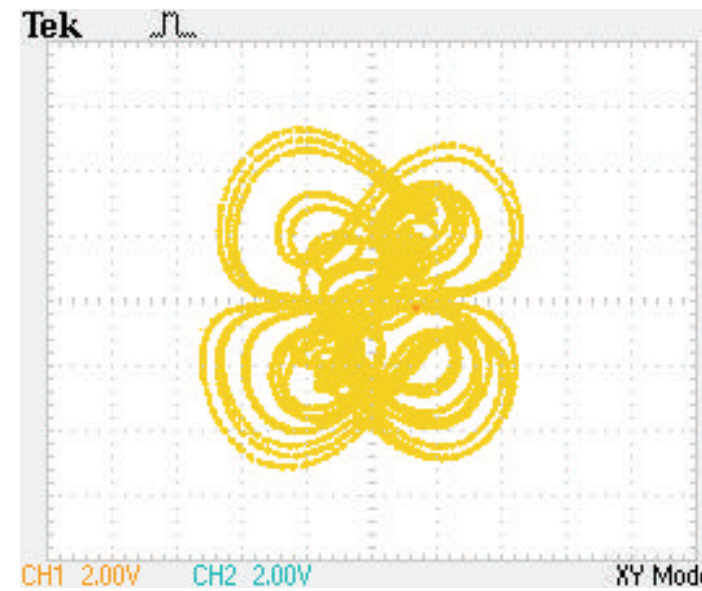

(d) Projection on the $x_{2}-x_{3}$ plane

FIGURE 15: Phase portrait of the real four-wing attractor by circuitry implementation.

\section{Circuit Experimental Researches}

In this part, an electronic oscillation circuit is designed to stabilize the equilibrium of the fractional-order four-wing hyperchaotic system based on inverse optimal control law.

As shown in Figure 11, the electronic oscillation circuit has two parts: one is the fractional-order four-wing hyperchaotic circuit and the other is an adaptive inverse optimal controller. The circuit consists of resistors, capacitors, operational amplifiers (AD741), and multipliers (AD633). The circuit parameters are as follows: $R_{1}=R_{3}=R_{9}=R_{14}=$ $100 \mathrm{k} \Omega, R_{2}=R_{4}=R_{8}=10 \mathrm{k} \Omega, R_{5}=R_{6}=R_{12}=1 \mathrm{k} \Omega$, $R_{13}=R_{18}=R_{19}=1 \mathrm{k} \Omega, R_{7}=R_{16}=40 \mathrm{k} \Omega, R_{10}=R_{11}=$ $R_{15}=10 \mathrm{k} \Omega, R_{17}=R_{20}=R_{22}=10 \mathrm{k} \Omega, R_{21}=2.5 \mathrm{k} \Omega$, $R_{23}=80 \mathrm{k} \Omega, R_{24}=400 \mathrm{k} \Omega, R_{25}=R_{27}=R_{29}=10 \mathrm{k} \Omega$, $R_{26}=400 \mathrm{k} \Omega, R_{28}=2 \mathrm{k} \Omega, R_{30}=20 \mathrm{k} \Omega, R_{31}=100 \mathrm{k} \Omega$, and $R_{32}=10 \mathrm{k} \Omega C_{5}=1 \mu \mathrm{F}$. State variables $x_{1}, x_{2}, x_{3}$, and $x_{4}$ are obtained from voltage outputs of fractional calculus unit, respectively. State feedback $e x_{4}$ is $R_{24}$ in a dotted line zone, which is the key of the four-wing system. The perturbation of parameter $b$ is generated by sine generator and multiplier.
To implement the electronic circuit of the fractionalorder system, the frequency domain approximation method is adopted. In [42], an effective algorithm is developed to approximate fractional-order transfer functions. From Table 1 in [14], one can obtain an approximation of $1 / s^{0.9}$ with an error of about $2 \mathrm{~dB}$ as follows:

$$
F(s)=\frac{2.2675(s+1.292)(s+215.4)}{(s+0.01292)(s+2.154)(s+359.4)} .
$$

The fractional calculus unit is shown in Figure 12, which is the chain fractance.

One can get the transfer function between $\mathrm{A}$ and $\mathrm{B}$. Consider

$$
G(s)=\frac{R_{a}}{1+R_{a} C_{a} s}+\frac{R_{b}}{1+R_{b} C_{b} s}+\frac{R_{c}}{1+R_{c} C_{c} s} .
$$

Using the method of undetermined coefficients and comparing (53) with (54), parameters of the fractional calculus unit are $R_{a}=62.84 \mathrm{M} \Omega, R_{b}=250 \mathrm{k} \Omega, R_{c}=2.5 \mathrm{k} \Omega, C_{a}=$ $1.232 \mu \mathrm{F}, C_{b}=1.835 \mu \mathrm{F}$, and $C_{c}=1.1 \mu \mathrm{F}$ [43]. 


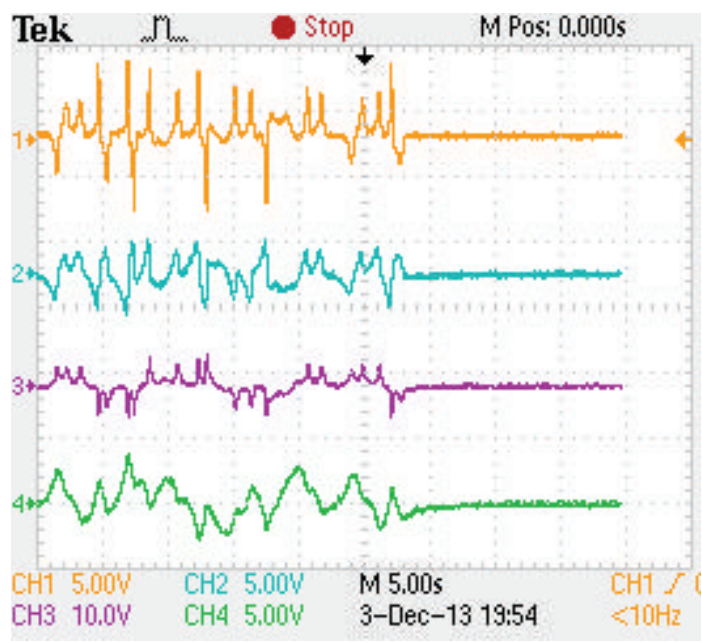

FIGURE 16: Time waveform of state variables of the controlled fractional-order system by circuitry implementation.

By circuit simulation, the real four-wing attractor without the controller is shown in Figure 13.

When $t=200 \mathrm{~s}$, the controller starts to work. As shown in Figure 14, state variables are closer to zero. In other words, the fractional-order four-wing hyperchaotic system is stabilized to its unstable equilibrium point.

Then an electronic oscillation circuit is constructed to implement the fractional-order four-wing attractor and the adaptive inverse optimal controller.

As shown in Figure 15, because the output of the circuit is limited precisely for using low-cost components, the experimental phase portraits of the fractional-order fourwing attractor are approximately in agreement with circuitry simulations.

The waveforms of state variables in time domain are shown in Figure 16. When the controller is added to the circuit, the waveforms are close to zero. It verifies the satisfactory performance of the proposed control law and proves the robustness of the system to some extent.

\section{Conclusions}

In this paper, combining the adaptive inverse optimal control with the stability theory of fractional-order system, a linear state feedback controller is designed to make the fractionalorder four-wing hyperchaotic system with uncertain parameter stable in the unstable equilibrium point. Through circuit simulations and circuit implementations, the method turned out to be workable. It is remarkably simple as comparing with other fractional chaos control methods which may have more than one nonlinear state feedback controller. This research has a certain theoretical and practical significance for the application of the adaptive inverse optimal control in nonlinear circuits, security communication, and many other engineering applications.

\section{Conflict of Interests}

The authors declare that there is no conflict of interests regarding the publication of this paper.

\section{Acknowledgment}

This paper is supported by the National Science Foundation for Post-doctoral Scientists of China (Grant no: 2013M530426).

\section{References}

[1] H.-Y. Jia, Z.-Q. Chen, and G.-Y. Qi, “Topological horseshoe analysis and circuit realization for a fractional-order Lü system," Nonlinear Dynamics, vol. 74, no. 1-2, pp. 203-212, 2013.

[2] D. Y. Chen, C. Wu, H. H. C. Iu, and X. Ma, "Circuit simulation for synchronization of a fractional-order and integer-order chaotic system," Nonlinear Dynamics, vol. 73, no. 3, pp. 16711686, 2013.

[3] N. Yang, C. Liu, and C. Wu, "Modeling and dynamics analysis of the fractional-order Buck-Boost converter in continuous conduction mode," Chinese Physics B, vol. 21, no. 8, Article ID 080503, 2012.

[4] S. Müller, M. Kästner, J. Brummund, and V. Ulbricht, "A nonlinear fractional viscoelastic material model for polymers," Computational Materials Science, vol. 50, no. 10, pp. 2938-2949, 2011.

[5] N. Laskin, "Fractional market dynamics," Physica A. Statistical Mechanics and Its Applications, vol. 287, no. 3-4, pp. 482-492, 2000.

[6] J. Y. Cao, C. B. Ma, Z. D. Jiang, and S. G. Liu, "Nonlinear dynamic analysis of fractional order rub-impact rotor system," Communications in Nonlinear Science and Numerical Simulation, vol. 16, no. 3, pp. 1443-1463, 2011.

[7] T. T. Hartley, C. F. Lorenzo, and H. K. Qammer, "Chaos in a fractional order Chua's system," IEEE Transactions on Circuits and Systems I: Fundamental Theory and Applications, vol. 42, no. 8, pp. 485-490, 1995.

[8] C. Li and G. Chen, "Chaos and hyperchaos in the fractionalorder Rössler equations," Physica A: Statistical Mechanics and Its Applications, vol. 341, no. 1-4, pp. 55-61, 2004.

[9] J. G. Lu and G. Chen, "A note on the fractional-order Chen system," Chaos, Solitons and Fractals, vol. 27, no. 3, pp. 685-688, 2006.

[10] J. G. Lu, "Chaotic dynamics of the fractional-order Lü system and its synchronization," Physics Letters A, vol. 354, no. 4, pp. 305-311, 2006.

[11] J. H. Lu, G. R. Chen, and D. Z. Cheng, "A new chaotic system and beyond: the generalized Lorenz-like system," International Journal of Bifurcation and Chaos in Applied Sciences and Engineering, vol. 14, no. 5, pp. 1507-1537, 2004.

[12] G. S. Hu, "A family of hyperchaotic systems with four-wing attractors," Acta Physica Sinica, vol. 58, no. 6, pp. 3734-3741, 2009.

[13] W. M. Ahmad and A. M. Harb, "On nonlinear control design for autonomous chaotic systems of integer and fractional orders," Chaos, Solitons and Fractals, vol. 18, no. 4, pp. 693-701, 2003.

[14] W. M. Ahmad and J. C. Sprott, "Chaos in fractional-order autonomous nonlinear systems," Chaos, Solitons and Fractals, vol. 16, no. 2, pp. 339-351, 2003. 
[15] C. P. Li and G. J. Peng, "Chaos in Chen's system with a fractional order," Chaos, Solitons and Fractals, vol. 22, no. 2, pp. 443-450, 2004.

[16] G. Qi, G. Chen, S. Li, and Y. Zhang, "Four-wing attractors: from pseudo to real," International Journal of Bifurcation and Chaos, vol. 16, no. 4, pp. 859-885, 2006.

[17] Z.-H. Wang, G.-Y. Qi, Y.-X. Sun, B. J. van Wyk, and M. A. van Wyk, "A new type of four-wing chaotic attractors in 3-D quadratic autonomous systems," Nonlinear Dynamics, vol. 60, no. 3, pp. 443-457, 2010.

[18] H. Li, X. Liao, and M. Luo, "A novel non-equilibrium fractionalorder chaotic system and its complete synchronization by circuit implementation," Nonlinear Dynamics, vol. 68, no. 1-2, pp. 137-149, 2012.

[19] S. T. Kingni, G. S. M. Ngueuteu, and P. Woafo, "Bursting generation mechanism in a three-dimensional autonomous system, chaos control, and synchronization in its fractionalorder form," Nonlinear Dynamics, vol. 76, no. 2, pp. 1169-1183, 2014.

[20] I. Grigorenko and E. Grigorenko, "Chaotic dynamics of the fractional Lorenz system," Physical Review Letters, vol. 91, Article ID 034101, 2006.

[21] A. S. Hegazi, E. Ahmed, and A. E. Matouk, "On chaos control and synchronization of the commensurate fractional order Liu system," Communications in Nonlinear Science and Numerical Simulation, vol. 18, no. 5, pp. 1193-1202, 2013.

[22] L. G. Yuan and Q. G. Yang, "Parameter identification and synchronization of fractional-order chaotic systems," Communications in Nonlinear Science and Numerical Simulation, vol. 17, no. 1, pp. 305-316, 2012.

[23] D. Chen, R. Zhang, J. C. Sprott, and X. Ma, "Synchronization between integer-order chaotic systems and a class of fractionalorder chaotic system based on fuzzy sliding mode control," Nonlinear Dynamics, vol. 70, no. 2, pp. 1549-1561, 2012.

[24] X. Y. Wang, X. P. Zhang, and C. Ma, "Modified projective synchronization of fractional-order chaotic systems via active sliding mode control," Nonlinear Dynamics, vol. 69, no. 1-2, pp. 511-517, 2012.

[25] D. Y. Chen, Y. X. Liu, X. Y. Ma, and R. F. Zhang, "Control of a class of fractional-order chaotic systems via sliding mode," Nonlinear Dynamics, vol. 67, no. 1, pp. 893-901, 2012.

[26] I. Podlubny, "Fractional-order systems and PI-lambda-D-mucontrollers," IEEE Transactions on Automatic Control, vol. 44, pp. 208-214, 1999.

[27] I. Podlubny, I. Petráš, B. M. Vinagre, and L. Dorčák, "Analogue realizations of fractional-order controllers," Nonlinear Dynamics, vol. 29, no. 1-4, pp. 281-296, 2002.

[28] S. E. Hamamci, "Stabilization using fractional-order PI and PID controllers," Nonlinear Dynamics, vol. 51, no. 1-2, pp. 329-343, 2008.

[29] O. P. Agrawal, "A general formulation and solution scheme for fractional optimal control problems," Nonlinear Dynamics, vol. 38, no. 1-4, pp. 323-337, 2004.

[30] N. Yang and C. Liu, "A novel fractional-order hyperchaotic system stabilization via fractional sliding-mode control," Nonlinear Dynamics, vol. 74, no. 3, pp. 721-732, 2013.

[31] M. J. R. Sepulchre and P. Kokotovic, Constructive Nonlinear Control, Springer, Santa Barbara, Calif, USA, 1997.

[32] H. K. Khalil and L. Praly, "High-gain observers in nonlinear feedback control," International Journal of Robust and Nonlinear Control, vol. 24, pp. 993-1015, 2014.
[33] G. H. Li, S. P. Zhou, and K. Yang, "Generalized projective synchronization between two different chaotic systems using active backstepping control," Physics Letters A: General, Atomic and Solid State Physics, vol. 355, no. 4-5, pp. 326-330, 2006.

[34] Y. Li, "A scheme of rössler chaotic synchronization under impulsive control," in Proceedings of the IITA International Conference on Services Science, Management and Engineering (SSME '09), pp. 472-474, Zhangjiajie, China, July 2009.

[35] K. S. Tsai, T. K. S. Shih, and T. S. Li, "New nonlinear controller for a class of chaotic systems based on adaptive backstepping fuzzy-immune control," Mathematical Problems in Engineering, vol. 2011, Article ID 439324, 20 pages, 2011.

[36] L. P. Liu, J. X. Pu, X. N. Song, Z. M. Fu, and X. H. Wang, "Adaptive sliding mode control of uncertain chaotic systems with input nonlinearity," Nonlinear Dynamics, vol. 76, no. 4, pp. 1857-1865, 2014.

[37] R. A. Freeman, Robust Nonlinear Control Design, Birkhäauser, Boston, Mass, USA, 1996.

[38] M. Krstic and P. Tsiotras, "Inverse optimal stabilization of a rigid spacecraft," IEEE Transactions on Automatic Control, vol. 44, no. 5, pp. 1042-1049, 1999.

[39] I. Podlubny, Fractional Differential Equations, Academic Press, San Diego, Calif, USA, 1999.

[40] D. Matignon, "Stability results for fractional differential equations with applications to control processing," in Proceedings of the Symposium on Control, Optimization and Supervision (CESA '96) and Multiconference: Computational Engineering in Systems Applications (IMACS '96), vol. 2, pp. 963-968, 1996.

[41] J. B. Hu, Y. Han, and L. D. Zhao, "A novel stability theorem for fractional systems and its applications in a synchronizing fractional chaotic system based on back-stepping approach," Acta Physica Sinica, vol. 58, no. 4, pp. 2235-2239, 2009.

[42] A. Charef, H. H. Sun, Y. Y. Tsao, and B. Onaral, "Fractal system as represented by singularity function," IEEE Transactions on Automatic Control, vol. 37, no. 9, pp. 1465-1470, 1992.

[43] C. X. Liu, "A hyperchaotic system and its fractional order circuit simulation," Acta Physica Sinica, vol. 56, pp. 6865-6873, 2007. 


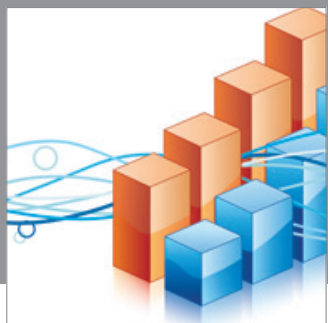

Advances in

Operations Research

mansans

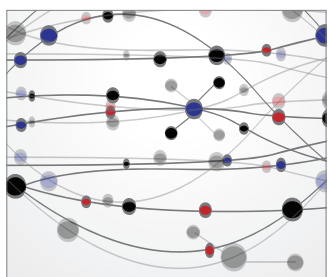

The Scientific World Journal
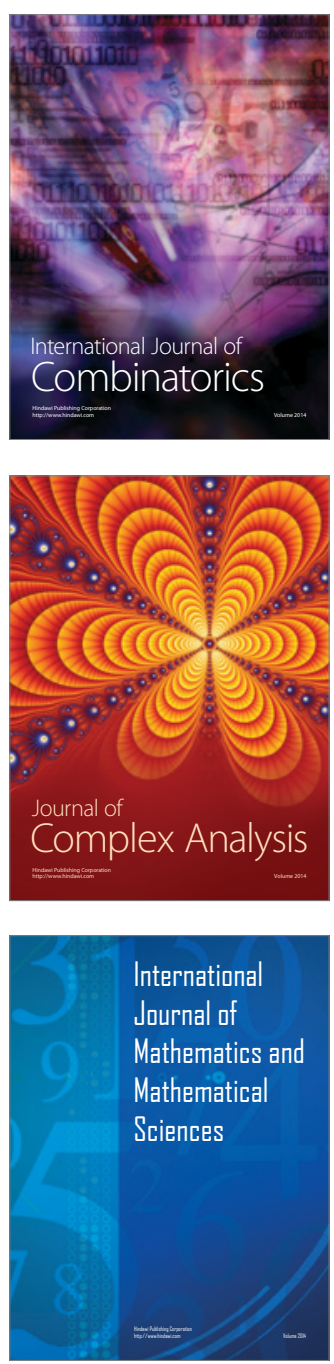
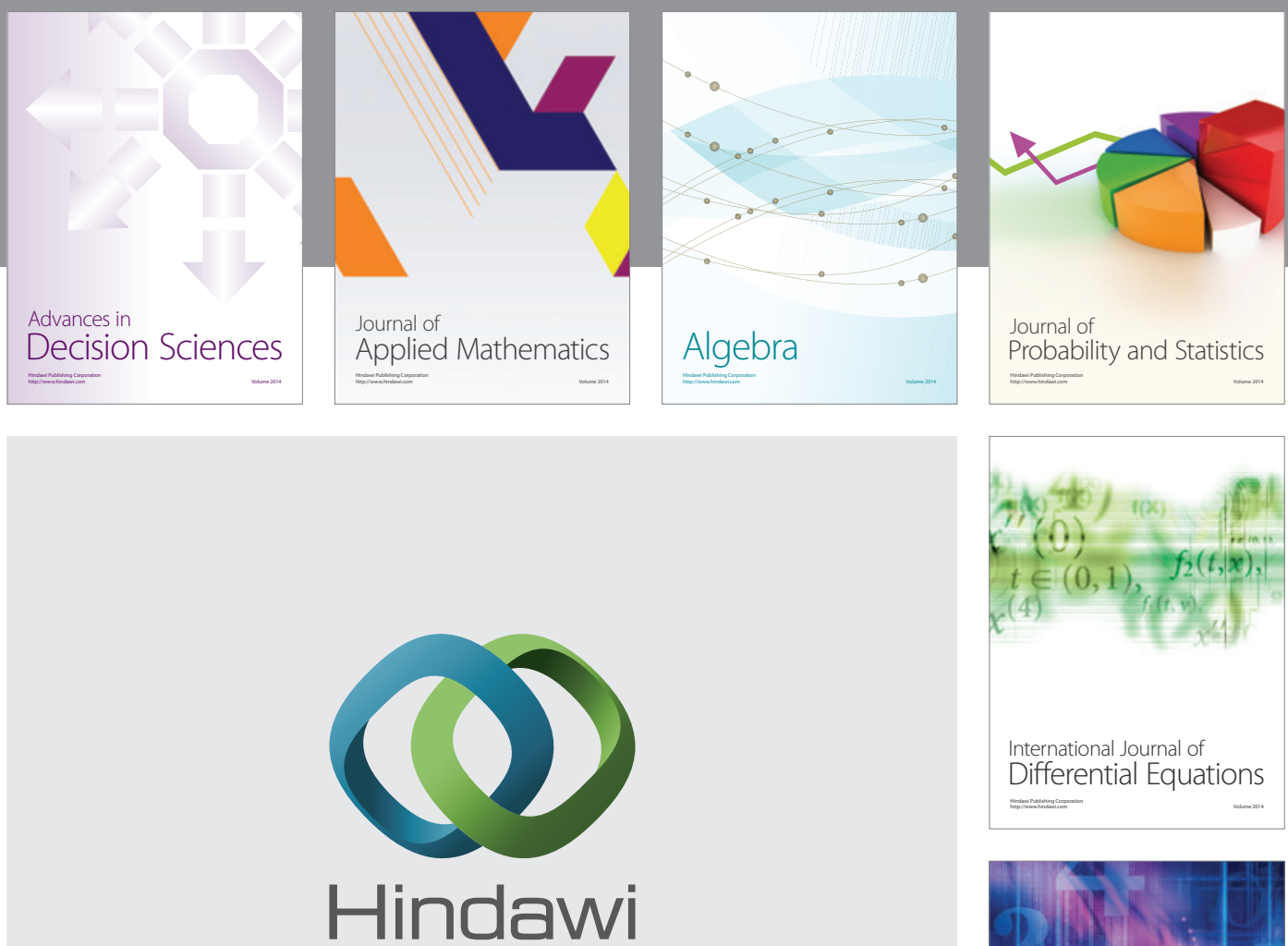

Submit your manuscripts at http://www.hindawi.com
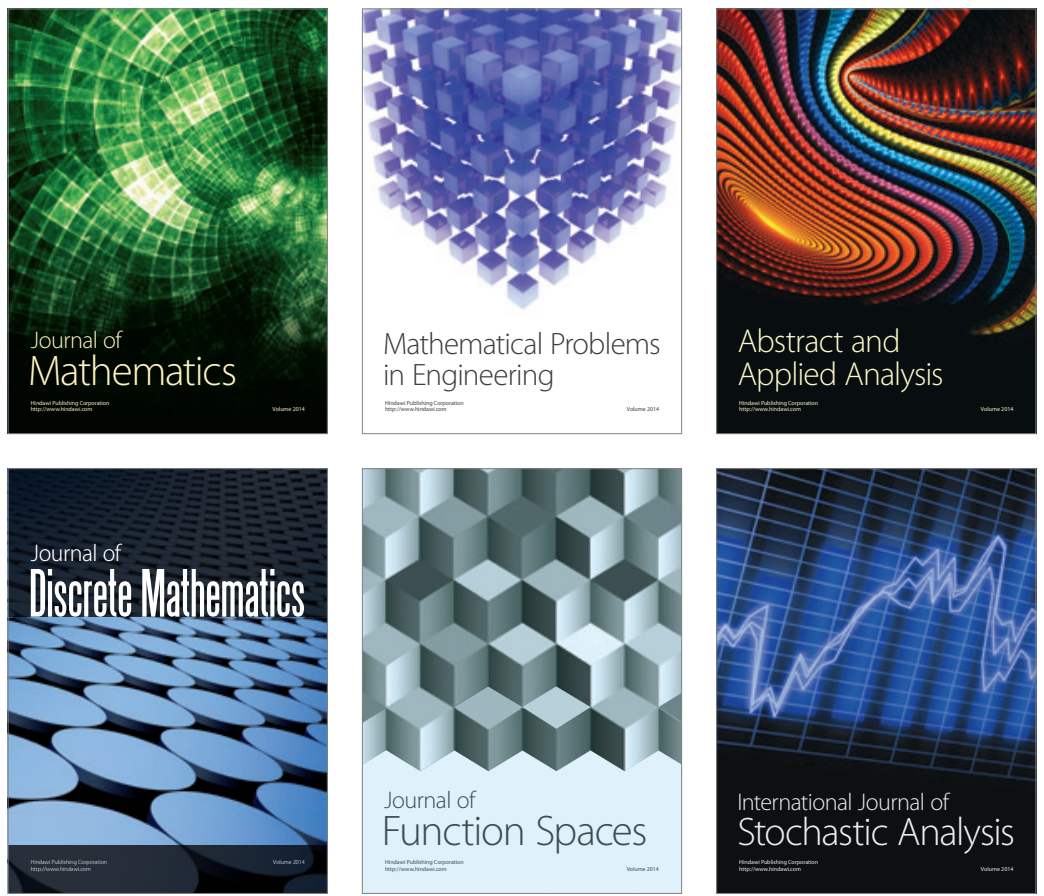

Journal of

Function Spaces

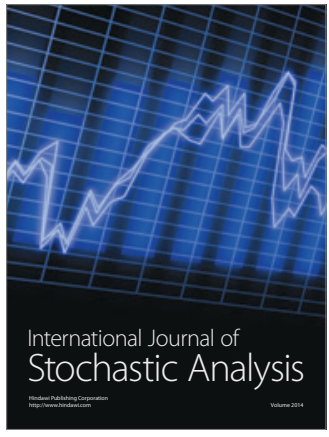

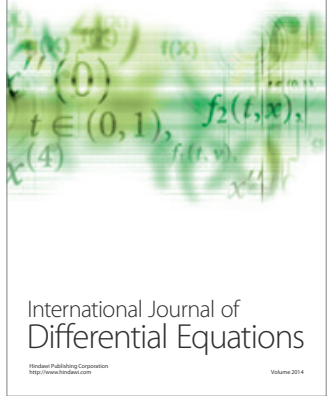
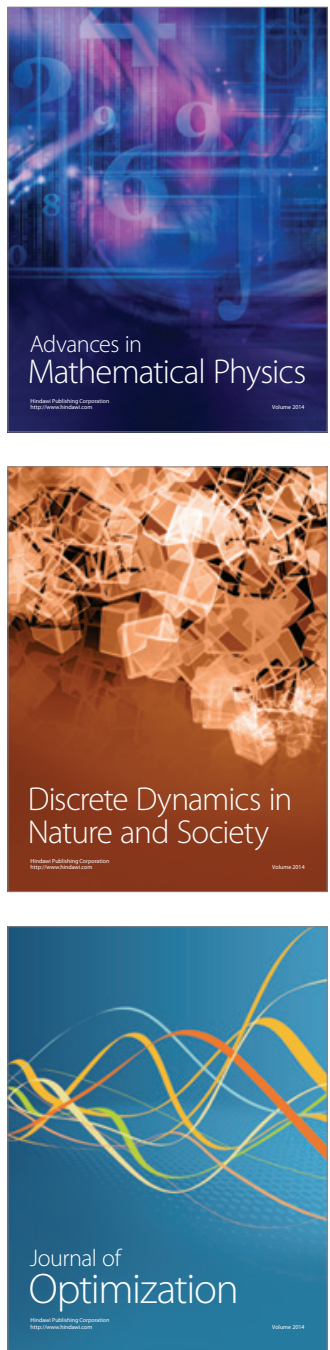